\title{
Quantum gauge models without (classical) Higgs mechanism
}

\author{
Michael Dütsch $^{1, a}$, José M. Gracia-Bondía ${ }^{2, b}$, Florian Scheck ${ }^{3, c}$, Joseph C. Várilly ${ }^{4, \mathrm{~d}}$ \\ ${ }^{1}$ Courant Research Center "Higher order structures in Mathematics", Mathematisches Institut, Univ. Göttingen, 37073 Göttingen, Germany \\ ${ }^{2}$ Departamento de Física Teórica, Universidad de Zaragoza, Zaragoza 50009, Spain \\ ${ }^{3}$ Institut für Physik, Theoretische Elementarteilchenphysik, Johannes Gutenberg-Universität, 55099 Mainz, Germany \\ ${ }^{4}$ Escuela de Matemática, Universidad de Costa Rica, San José 2060, Costa Rica
}

Received: 14 June 2010 / Revised: 19 August 2010 / Published online: 15 September 2010

(C) Springer-Verlag / Società Italiana di Fisica 2010

\begin{abstract}
We examine the status of massive gauge theories, such as those usually obtained by spontaneous symmetry breakdown, from the viewpoint of causal (EpsteinGlaser) renormalization. The BRST formulation of gauge invariance in this framework, starting from canonical quantization of massive (as well as massless) vector bosons as fundamental entities, and proceeding perturbatively, allows one to rederive the reductive group symmetry of interactions, the need for scalar fields in gauge theory, and the covariant derivative. Thus the presence of higgs particles is understood without recourse to a Higgs(-Englert-BroutGuralnik-Hagen-Kibble) mechanism. Along the way, we dispel doubts about the compatibility of causal gauge invariance with grand unified theories.
\end{abstract}

$$
\begin{array}{r}
\text { "Sire, je n'avais pas besoin de cette hypothèse-là" } \\
\text { - Pierre Simon de Laplace }
\end{array}
$$

\section{Introduction}

With the start of the LHC operation, the Higgs sector of the Standard Model (SM) and Higgs' mechanism of spontaneous symmetry breakdown (SSB) allegedly giving rise to it [1-4] have become a topical issue [5].

The Higgs sector has unsatisfactory aspects, often discussed: the self-coupling terms appear to be ad-hoc, unrelated to other aspects of the theory, not seeming to constitute a gauge interaction [6, Sect. 22]. They raise the hierarchy problem [7, 8]. Since most fields involved in the Higgs

\footnotetext{
a e-mail: michael.duetsch@theorie.physik.uni-goettingen.de

be-mail: jmgb@unizar.es

c e-mail: scheck@uni-mainz.de

d e-mail: joseph.varilly@ucr.ac.cr
}

mechanism are unobservable, the status question for it cannot be simply resolved by sighting of higgses ${ }^{1}$ in the LHC. Arguably this is why, at the end of his Nobel lecture, Veltman wrote: "While theoretically the use of spontaneous symmetry breakdown leads to renormalizable Lagrangians, the question of whether this is really what happens in Nature is entirely open" [9]. This mistrust, also apparent in [10], is more widespread than current orthodoxy would have us believe. The theoretical puzzles, as well as present phenomenological ones, advise a new look at the scalar sectors of the SM and grand unified theories (GUTs) within a quantum field theoretic framework.

In this introduction we first briefly summarize experimental information on the Higgs sector of the standard model. Then the conclusions of the "reality check" on the Higgs mechanism worked out in this paper are compared to the ones of a first reality check performed in the midseventies. Finally we summarize the contents of the paper.

\subsection{Phenomenological puzzles}

Generally speaking, precision electroweak measurements were successful in pinning down new features of the SM and its constituents, even before actual discoveries took place. Perhaps the best example is provided by the top quark, whose mass could be estimated rather precisely from a global analysis of all available electroweak data before it was found at Fermilab. To illustrate this point we quote a global fit of SM electroweak data [11], excluding the directly measured top mass, which yields $178.2_{-4.2}^{+9.8} \mathrm{GeV}$. If the experimental value $172.4_{-1.2}^{+1.2} \mathrm{GeV}$ is included, one obtains the improved fit value $172.9_{-1.2}^{+1.2} \mathrm{GeV}$, not too different from the indirect determination.

\footnotetext{
${ }^{1}$ Following L.B. Okun, and for obvious grammatical reasons, we refer to a (physical) Higgs boson as higgs, with a lower-case $h$.
} 
The case of the Higgs boson at present is more complicated. The result of the standard fit not taking into account direct searches for the higgs (i.e. the lower limits on the higgs mass $M_{\mathrm{H}}$ obtained at LEP and the Tevatron) is:

$M_{\mathrm{H}}=80_{-23}^{+30} \mathrm{GeV}$.

The complete fit of all data, including the lower limits, gives the estimate

$M_{\mathrm{H}}=116.4_{-1.3}^{+18.3} \mathrm{GeV}$.

The deviation of the central values in (1) and (2) from one another are due to contradictory tendencies in the data. Most notable among these is the forward-backward asymmetry $A_{\mathrm{FB}}^{0, b}$ into $b$-quarks whose pull value in the complete fit is

$\frac{1}{\sigma_{\text {meas }}}\left(\left.A_{\mathrm{FB}}^{0, b}\right|_{\text {fit }}-\left.A_{\mathrm{FB}}^{0, b}\right|_{\text {meas }}\right)=2.44$,

the denominator $\sigma_{\text {meas }}$ being the error in the measurement. This hadronic asymmetry, taken in isolation, yields a tendency to rather high values of the higgs mass, while the leptonic asymmetries in the case of the LEP data either agree with the value (2) or tend towards lower values of that mass as is the case for the SLD data-consult Fig. 3 in [11]. In spite of the tension between leptonic and hadronic asymmetries, no single pull value exceeds the $3 \sigma$ level. The present state of such fits, and the influence of the (more than twenty) SM quantities on them, are well summarized in Table 1 and Figs. 2 and 3 of [11]. The divergent influences of various electroweak data on the mass of the higgs were noted already a few years ago [12]. They have been quantified in [13], emphasizing the strong correlation between $A_{\mathrm{FB}}^{(0, b)}$ and the predicted higgs mass.

The modest quality of the overall fit might be due to inconsistencies in the data and/or radiative corrections, that might disappear when further progress is made. However, it might as well signal that the scalar sector is considerably more complicated than in standard lore, leading to a reduction of the standard higgs couplings. Consequently something could have been overlooked at LEP: for instance, mixing with "hidden world" scalars [14] yields such a reduction, in particular for the ZZH coupling; and this could not be, and was not, ruled out by LEP2 for those relatively low energies. Other scenarios shielding the higgs from detection have been discussed in the literature-see $[15,16]$ as well as the illuminating remarks in [17]. Recent experiment has made the situation even murkier: on Halloween night of 2008, ghostly (albeit rather abundant) multi-muon events at Fermilab were reported by the CDF collaboration [18, 19]. A possible explanation for them invokes new light higgs-like particles coupling relatively strongly to the "old" ones, and less so to the SM fermions and vector bosons [20,21]. Strassler has cogently emphasized that "minimality" of the scalar sector of the SM is just a theoretical prejudice [22]. (Yes, entia non sunt multiplicanda praeter necessitatem. But, who ordered the muon?) The recent discovery of excess charge asymmetry (that is $\mathrm{CP}$ violation) in $b$-hadrons [23] points in the same direction [24]. Given this state of affairs, it seems premature to draw any definitive conclusion.

\subsection{Reality checks for SSB}

Causal perturbation theory as developed by $\mathrm{H}$. Epstein and V. Glaser (EG) and applied to QED by Scharf and collaborators $[25,26]$ does not look applicable a priori to nonAbelian gauge theories. Indeed the EG method involves an expansion in terms of the coupling constant(s) whereas, as is well known, gauge invariance in the non-Abelian case interrelates terms of different orders in these couplings. Nevertheless, causal gauge invariance (CGI) interprets BRST symmetry as a fundamental property of quantum gauge theory-in the spirit of [27] and [28, Sect. 3.3], providing a canonical description of vector bosons, eliminating unphysical fields, and helping (through the consistency relations it imposes) to reconstruct the gauge-invariant Lagrangian from a general Ansatz.

In this context it may be useful to recall a half-forgotten chapter of the early history of gauge theory, chiefly due to Bell, Cornwall, Levin, Llewelyn Smith, Sucher, Tiktopoulos and Woo in the seventies: see [29, 30] and references therein. The connection between "tree-unitarity" (the natural high-energy boundedness condition for $\mathbb{S}$-matrix elements in the tree approximation) and perturbative unitarity, leading to plausible renormalizability requisites for Lagrangians, was understood by then. All those papers started essentially without preconditions from Lagrangians made out of massive vector bosons (MVB) as fundamental entities, and found that:

- First and foremost, the couplings of the vector bosons had to be of the gauge theory type, governed by reductive symmetry groups (as in physics parlance, "groups" often denote "Lie algebras" in this paper).

- Furthermore, scalar fields necessarily entered the picture. The allowed theories so obtained were essentially equivalent to (the phenomenological outcome of) SSB models, with one general exception: "Abelian mass terms" were possible for the vector bosons.

The latter is understandable: QED with massive photons is a well-behaved theory. Whenever the symmetry group possesses an invariant Abelian subgroup, one may add such terms. For our purposes this second finding is not moot, since it suggests the description of spin-1 massive models with the help of Stückelberg fields. After all, the SM contains an invariant Abelian subgroup and the mass of the $Z$ 
particle can be (though it need not be) thought to be of that type, see the discussion in Appendix A. Stückelberg fields are of course unphysical. But they have a rightful place in quantum field theory for the canonical description of MVB, already at the level of free fields [31-34].

Right afterwards the BRST revolution took hold, and the formalism for gauge symmetry changed forever. ${ }^{2}$

\subsection{Outline of the sequel}

The book by Scharf [35], crowning a successful line of research [36-43] which in particular establishes a consistent formulation of the SM without SSB [41], aimed to bring a fresh perspective to the subject from the standpoint of CGI. In tune with it, with the earlier reality check, and with the phenomenological SM Lagrangian, here we stop pretending we know the origin of mass, and start without preconditions again from MVB as fundamental fields. That the reductive Lie algebra structure then follows from CGI was recognized by Stora in [44], which constituted an important motivation for this work.

In Sect. 2 we expose the theoretical underpinnings of our own reality check. There are actually at least two CGI methods; both are expounded there. The first method is constructive. The second, stemming from a theorem by one of us in [45], is useful rather to verify CGI.

Section 3 summarizes the first results of the theory. We report the outcome of that first method, rendering the cubic coupling relations for CGI models, determined by BRST invariance at first and second order.

The work on tree-unitarity invoked above seemed to certify every SSB-kind model as acceptable. On the other hand, Ambauen and Scharf have claimed [46] that the CGI approach clashes with the outcome of SSB for the GeorgiGlashow GUT. The matter deserved further investigation, all the more so since their assertion is in contradiction with the second CGI method. As it turns out, CGI produces constraints on the allowed patterns of masses and couplings. A certain obstruction put forward by Scharf, sensible enough in some circumstances, was responsible for the rejection of the Georgi-Glashow $S U(5)$ and other scenarios. Next in Sect. 4 we unravel this internal problem in CGI by uncovering an oversight responsible for the mentioned rejections: there is no problem with GUTs.

Properly reformulated, the obstruction is the germ of the general $S$-representation, that is, of a derivation from first principles of the covariant derivative coupling, familiar in the standard approaches. The theorem in [45] of course fits with our construction. The previous analysis allows one next

\footnotetext{
${ }^{2}$ Some of the authors of that reality check openly suspected SSB as a formal recipe without physical meaning; others were swayed by the remarkable "success rate" of the Higgs mechanism; some apparently remained agnostic. And so is the case with the present writers.
}

to describe what is presently known to us on quartic terms in the Lagrangian from CGI.

Section 5, intended to familiarize the reader thoroughly with the workings of CGI, is made out of examples. Some readers might prefer to go to this section before tackling the general aspects expounded before. First we review an Abelian model. Next we examine a few slightly more complicated models within CGI. We put aside the Abelian exception by dealing with simple groups; this dictates the number of vector bosons (corresponding to Cartan's classical groups) for irreducible symmetry realizations. We consider allowed mass patterns for models with one higgs, pondering first the simple but all-important case with only three gauge bosons, and next CGI for higher-rank groups. We recall the corresponding SSB mindset: the choice of only one higgs corresponds to mass patterns produced by the Higgs mechanism by vector realizations of the gauge group. Then we look at BRST invariance and minimal coupling from CGI corresponding to SSB with fields in the adjoint representation.

Section 6 dwells on our conclusions.

To put matters in perspective, in Appendix A we report on the SM from the angle of CGI. ${ }^{3}$ Some technical aspects of the machinery underlying this work are confined to Appendix B. Finally in Appendix $\mathrm{C}$ we amplify on the epistemological implications of the article.

\section{The scheme of causal gauge invariance}

\subsection{The method in general}

In the origin of causal perturbation theory [47], the formulation of gauge symmetry and its preservation in the process of renormalization was not taken into account. Besides other related methods to deal with symmetries in that framework [48-51], causal gauge invariance is a systematic technique to treat quantum gauge theories perturbatively by EpsteinGlaser renormalization. As pointed out above, it was first broached by Scharf and collaborators for QED. A developed formulation was found in the treatment of massless Yang-Mills theories [36, 37]. It has been applied successfully also to massive non-Abelian models, namely the SM [41, 42], spin-2 gauge fields and supersymmetry. Quite recently, the method has been recruited to examine the issue of the (putative) decoupling of ghosts in a class of noncovariant gauges [52].

Rather than follow the motivation of CGI in the books [26] and [35], we adopt a viewpoint inspired by (perturbative) algebraic quantum field theory. BRST invariance is input already in the canonical description of vector bosons.

\footnotetext{
${ }^{3}$ We regret that its elegant formulation without SSB is so widely ignored.
} 
The unphysical fields are eliminated by using the BRST transformation $s$ : the algebra of observables is obtained as its cohomology, implemented by the nilpotent BRST charge $Q$. The space of physical states can be described cohomologically as well-see in particular [53].

The construction of $Q$ in perturbative gauge field theory meets the problem that in general the BRST charge $Q$ changes when the interaction is switched on [53]. For theories with good infrared behavior like purely massive theories, Kugo and Ojima [54] showed that $Q$ can be identified with the incoming (free) BRST charge $Q_{\text {in }}$, which implements the BRST transformation $s_{0}$ of the incoming fields. That the $\mathbb{S}$-matrix be well-defined on the physical Hilbert space of the free theory amounts to the requirement $[31,43]$ :

$\left.\lim _{g \uparrow 1}\left[Q_{\text {in }}, \mathbb{S}\left(g \kappa L_{1}\right)\right]\right|_{\text {ker } Q_{\text {in }}}=0$.

Here $\mathbb{S}\left(g \kappa L_{1}\right)$ is the $\mathbb{S}$-matrix corresponding to the interaction $g(x) \kappa L_{1}(x)$, an operator in the Fock space of the incoming free fields. The local Wick polynomial $L_{1}$ is the part of the total interaction Lagrangian $L_{\text {tot }}=\sum_{n=1}^{\infty} \kappa^{n} L_{n}$ linear in the coupling constant $\kappa$ (notationally assumed unique for simplicity). The function $g \in \mathcal{S}\left(\mathbb{R}^{4}\right)$ switches the coupling constant on and off; the adiabatic limit $g \uparrow 1$ has to be performed to obtain the physically relevant $\mathbb{S}$-matrix. Now, $\mathbb{S}\left(g \kappa L_{1}\right)$ is a formal power series,

$$
\begin{aligned}
\mathbb{S}\left(g \kappa L_{1}\right)=\mathbf{1} & +\sum_{n \geq 1} \frac{i^{n} \kappa^{n}}{n !} \int d x_{1} \cdots d x_{n} g\left(x_{1}\right) \cdots g\left(x_{n}\right) \\
& \times T_{n}\left(L_{1}\left(x_{1}\right) \cdots L_{1}\left(x_{n}\right)\right),
\end{aligned}
$$

where the time-ordered product $T_{n}\left(L_{1}\left(x_{1}\right) \cdots L_{1}\left(x_{n}\right)\right)$ is an operator-valued distribution.

The higher-order terms of the interaction $L_{n}$ for $n \geq 2-$ which are also local Wick polynomials - are taken into account as local terms in $T_{n}\left(L_{1}\left(x_{1}\right) \cdots L_{1}\left(x_{n}\right)\right)$ : the latter will contain a term

$n !(-i)^{n-1} \delta\left(x_{1}-x_{n}, \ldots, x_{n-1}-x_{n}\right) L_{n}\left(x_{n}\right)$,

which propagates to higher orders $n^{\prime}>n$ by the inductive machinery of Epstein-Glaser renormalization [25].

To satisfy (3) to first order in $\kappa$, one just searches for a local Wick polynomial $P_{v}$ (called a " $Q$-vertex") such that

$s_{0} L_{1}(x) \equiv\left[Q_{\text {in }}, L_{1}(x)\right]=\partial^{v} P_{\nu}(x)$.

Turning to higher orders, we first note that if $x_{i} \neq x_{j}$ for all $i<j$, there is a permutation $\pi$ such that $x_{\pi(j)} \cap\left(x_{\pi(j+1)}+\right.$ $\left.V_{-}\right)=\emptyset$ for every $j$, with $V_{-}$being the solid backward lightcone. Hence, for such configurations the time-ordered product can be written as a standard operator product:

$T_{n}\left(L_{1}\left(x_{1}\right) \cdots L_{1}\left(x_{n}\right)\right)=L_{1}\left(x_{\pi 1}\right) \cdots L_{1}\left(x_{\pi n}\right)$.
In view of

$$
\begin{aligned}
& {\left[Q_{\text {in }}, L_{1}\left(x_{1}\right) \cdots L_{1}\left(x_{n}\right)\right]} \\
& =\sum_{l=1}^{n} L_{1}\left(x_{1}\right) \cdots\left[Q_{\text {in }}, L_{1}\left(x_{l}\right)\right] \cdots L_{1}\left(x_{n}\right) \\
& =\sum_{l=1}^{n} \partial_{x_{l}}^{v}\left(L_{1}\left(x_{1}\right) \cdots P_{v}\left(x_{l}\right) \cdots L_{1}\left(x_{n}\right)\right),
\end{aligned}
$$

one generalizes (5) to higher orders by requiring that

$$
\begin{aligned}
s_{0} & T_{n}\left(L_{1}\left(x_{1}\right) \cdots L_{1}\left(x_{n}\right)\right) \\
& \equiv\left[Q_{\mathrm{in}}, T_{n}\left(L_{1}\left(x_{1}\right) \cdots L_{1}\left(x_{n}\right)\right)\right] \\
& =\sum_{l=1}^{n} \partial_{x_{l}}^{v} T_{n}\left(L_{1}\left(x_{1}\right) \cdots P_{\nu}\left(x_{l}\right) \cdots L_{1}\left(x_{n}\right)\right) .
\end{aligned}
$$

Formulas (5) and (7) constitute the operator CGI conditions, enough to guarantee (3) if the adiabatic limit exists. (In theories involving massless fields that limit is problematic, to be sure. For instance, in QED the $\mathbb{S}$-matrix contains infrared divergences, which cancel in the cross sections. In models with confinement, the situation is worse, and a perturbative treatment is possible only for short distances; an adequate description is the local construction of the observables [53] by using couplings $g(x) \kappa$ with a compactly supported test function $g$. However, the CGI conditions (5) and (7) are well defined even in models with bad infrared behavior; in that case they can be justified by deriving them from the conservation of the BRST current for non-constant coupling $[49,50]$.)

Requirement (7) is a renormalization condition, which restricts also tree diagrams, see below. Namely, if the sequence of time-ordered products $\left\{T_{n}\right\}$ is constructed inductively by causal perturbation theory [47], from (6) we conclude that CGI can be violated only in the extension to the total diagonal $\Delta_{n} \equiv\left\{\left(x_{1}, \ldots, x_{n}\right) \in \mathbb{R}^{4 n}: x_{1}=\cdots=x_{n}\right\}$ of the $T_{n}$; that is, the extension from $\mathcal{S}^{\prime}\left(\mathbb{R}^{4 n} \backslash \Delta_{n}\right)$ to $\mathcal{S}^{\prime}\left(\mathbb{R}^{4 n}\right)$ at the level of numerical distributions. Indeed there is violation, in that causal splitting does not respect the divergences in general; however, CGI can be restored. That gauge-invariant causal renormalization can be performed to all orders has been proved for QED [26] and massless $S U(N)$ Yang-Mills theories [36-39]. ${ }^{4}$

\footnotetext{
${ }^{4}$ One expects that the only obstructions to CGI stem from the usual anomalies of quantum field theory. Our general analysis includes all gauge models which are known to be free of anomalies; but there are cases where CGI at tree level applied to a general Ansatz for the Lagrangian generates a model exhibiting anomalies; this happens for the axial anomaly [42]. The question may be examined quite generally by algebraic criteria developed in [55, 56] and [51]. The former authors showed in particular that gauge-invariant and gauge-fixed cohomologies in the EG framework are equivalent.
} 
In summary, within CGI determination by BRST cohomology acts as a subsidiary physical rule. This answers to the deeply rooted [57] need to amend Wigner representation theory of particles for bosons with spin 1 in Fock space with a Krein structure. Recognition of this is an undoubted merit of [35]. (Cherished positivity could be restored at the price of nonlocality; behind the veil, string-localized potentials and new field theory phases might well lurk [58, 59].) We aver that CGI and the addition of higgs-like fields (in the next subsection) is only a sufficient and not a necessary condition for unitarity - consult [38, 39] and [60] in this respect. A non-perturbative understanding of field theory could restore unitarity in some other way. However, in appropriate contexts and hands, perturbative methods have something to say about non-perturbative issues. An example is provided by the exploitation of perturbative BRST invariance in the understanding of confinement [61] - recently, the same approach has been applied by Nishijima and Tureanu to the study of the gauge dependence of the Green's functions in non-Abelian gauge theory [62].

\subsection{The grubby machinery}

As said above, (5) and (7) are already nontrivial when used for tree diagrams. CGI strongly restricts the set of allowed models, determining the interaction $L=\sum_{n=1}^{\infty} \kappa^{n} L_{n}$ to a great extent, independently of the infrared behavior. Given the free theory, one makes a polynomial and renormalizable Ansatz for $L_{1}$. The CGI condition (5) determines most of the coefficients in this Ansatz, or yields relations between them. Turning to higher-order tree diagrams, terms of the form (4) remain undetermined in the inductive Epstein-Glaser construction. Then (7) determines the higher-order interaction terms $L_{n}$ and the as yet undetermined coefficients of $L_{1}$. The process is constructive. The reductive Lie algebraic structure and the need to add additional physical scalar fields (higgs fields) in massive non-Abelian models are not to be put in; they follow from CGI. To be precise in the last respect: for such a model with MVB and only the unphysical fermionic ghosts and Stückelberg fields, CGI breaks down at order $\kappa^{2}$ [41]; however the inclusion of at least one additional physical scalar makes CGI solvable. The "puzzling" [63, Preface] existence of fundamental scalars is demanded in our framework.

The process terminates after a finite number of steps in renormalizable theories. Consider spin-1 gauge models in 4-dimensional Minkowski space with an $L_{1}$ trilinear in the fields whose mass dimension is $\leq 4$. Then tree diagrams can give nontrivial constraints only up to third order. Indeed, because CGI can be violated only in the extension of $T_{n}$ to $\Delta_{n}$, a possible violation of (7) must be of the form

$\sum_{a, \mathcal{O}} C_{a, \mathcal{O}} \partial^{a} \delta\left(x_{1}-x_{n}, \ldots, x_{n-1}-x_{n}\right) \mathcal{O}\left(x_{1}, \ldots, x_{n}\right)$, where $\mathcal{O}\left(x_{1}, \ldots, x_{n}\right)$ denotes a normally ordered product of free fields, $a=\left(a_{l}^{\mu}\right)_{l=1, \ldots, n-1}^{\mu=0,1,2,3}$ is a multi-index and the $C_{a, \mathcal{O}}$ are suitable numbers. Power counting yields the restriction $|a|+\operatorname{dim} \mathcal{O} \leq 5$ with $|a| \equiv \sum_{l, \mu} a_{l}^{\mu}$. Since each vertex of $L_{1}$ has three legs, it follows that a tree diagram to $n$-th order satisfies $\operatorname{dim} \mathcal{O} \geq 2+n$. Combining these two inequalities, one sees that CGI can be violated at tree level only for $n \leq 3$. In practice, most interesting information is concentrated at the first and second orders; third-order CGI only refines a few coefficients in the Higgs sector.

We illustrate the construction of the time-ordered products at the tree level at second order, in the case of spin-1 gauge fields, following essentially [41]; the bulk of calculations buttressing this paper are of this type. Note

$$
\begin{aligned}
& \left.T_{2}(x, y)\right|_{\text {tree }}:=\left.T_{2}\left(L_{1}(x) L_{1}(y)\right)\right|_{\text {tree }}, \\
& \left.T_{2 / 1}^{v}(x, y)\right|_{\text {tree }}:=\left.T_{2}\left(P^{v}(x) L_{1}(y)\right)\right|_{\text {tree }},
\end{aligned}
$$

as well as $\left.T_{2 / 2}^{v}(x, y)\right|_{\text {tree }}=\left.T_{2 / 1}^{v}(y, x)\right|_{\text {tree }}$, can be written as

$$
\left.T_{2}\right|_{\text {tree }}=\left.T_{2}\right|_{\text {tree }} ^{0}+N_{2},\left.\quad T_{2 / 1}^{v}\right|_{\text {tree }}=\left.T_{2 / 1}^{v}\right|_{\text {tree }} ^{0}+N_{2 / 1}^{v},
$$

where $\left.T_{2}\right|_{\text {tree }} ^{0},\left.T_{2 / 1}^{v}\right|_{\text {tree }} ^{0}$ include all terms not vanishing for $x \neq y$; these are the terms with the Feynman propagator $\Delta_{m}^{F}$ or its derivatives $\partial_{\mu} \Delta_{m}^{F}, \partial_{\nu} \partial_{\mu} \Delta_{m}^{F}$. We replace $\square \Delta_{m}^{F}$ by $-m^{2} \Delta_{m}^{F}+\delta$; the $-m^{2} \Delta_{m}^{F}$ term belongs to $\left.T_{2}\right|_{\text {tree }} ^{0}$ and the $\delta$-term to $N_{2}$. Expressions $N_{2}$ and $N_{2 / 1}$ are of the form

$N_{2}(x, y)=\kappa^{2} \sum_{\phi_{1} \phi_{2} \phi_{3} \phi_{4}} C_{\phi_{1} \phi_{2} \phi_{3} \phi_{4}} \delta(x-y): \phi_{1} \phi_{2} \phi_{3} \phi_{4}(x)$ :

and similarly for $N_{2 / 1}$, where $C_{\phi_{1} \phi_{2} \phi_{3} \phi_{4}}$ are $c$-numbers, and the sum runs over all kinds of free fields $\phi_{1}, \ldots, \phi_{4}$ present in the model. In the framework of causal perturbation theory, such local terms may be added to $T_{2}$, if they respect power counting, Lorentz covariance, unitarity, ghost number, etc. A glance at formula (4) indicates that $N_{2}(x, y)=$ $-2 i \delta(x-y) L_{2}(x)$, where $L_{2}$ is a sum of quartic terms.

In contrast to $\left.T_{2}\right|_{\text {tree }} ^{0}$ and $\left.T_{2 / 1}^{v}\right|_{\text {tree }} ^{0}$, which are uniquely given in terms of $L_{1}$ and $P^{v}$, the coefficients in $N_{2}$ and $N_{2 / 1}$ are not yet determined. To prove CGI for second-order tree diagrams, we have to show that the as yet undetermined coupling parameters of $L_{1}$ and the coefficients $C_{\phi_{1} \phi_{2} \phi_{3} \phi_{4}}$ in $N_{2}$ and $N_{2 / 1}$ can be chosen in such a way that

$$
\begin{aligned}
& {\left[Q_{\text {in }},\left(\left.T_{2}\right|_{\text {tree }} ^{0}+N_{2}\right)(x, y)\right]} \\
& \quad=\partial_{v}^{x}\left(\left.T_{2 / 1}^{v}\right|_{\text {tree }} ^{0}+N_{2 / 1}^{v}\right)(x, y)+[x \leftrightarrow y] .
\end{aligned}
$$

Since this condition holds by induction for $x \neq y$, we need only study the local contributions. However, the splitting of a distribution into local and nonlocal parts in principle is not unique, and some caution is called for. Recall that we 
replace (כ) $\square \Delta_{m}^{F}$ by $-m^{2}(\partial) \Delta_{m}^{F}+(\partial) \delta$. Then for $x \neq y$ only terms $\sim \Delta_{m}^{F}, \sim \partial_{\mu} \Delta_{m}^{F}, \sim \partial_{\nu} \partial_{\mu} \Delta_{m}^{F}$ and $\sim \partial_{\nu} \partial_{\mu} \partial_{\lambda} \Delta_{m}^{F}$ with no contraction of Lorentz indices contribute to (8). Since these terms cancel for $x \neq y$, they cancel for $x=y$ also. There remain only terms $\sim \delta(x-y)$ and $\sim \partial \delta(x-y)$. For spin-1 gauge theories, such $(\partial) \delta$-terms can be generated only in the following ways.

1. First of all,

$$
\begin{aligned}
& {\left[Q_{\text {in }}, N_{2}(x, y)\right]} \\
& =\kappa^{2} \sum_{\phi_{1} \phi_{2} \phi_{3} \phi_{4}} C_{\phi_{1} \phi_{2} \phi_{3} \phi_{4}} \delta(x-y) \\
& \quad \times\left(:\left[Q_{\text {in }}, \phi_{1}(x)\right] \phi_{2}(x) \cdots:\right. \\
& \left.\quad+: \phi_{1}(x)\left[Q_{\text {in }}, \phi_{2}(x)\right] \cdots:+\cdots\right) .
\end{aligned}
$$

2. From $N_{2 / 1}^{v}(x, y)=C^{v} \delta(x-y) M(x)$, where $M=$ $: \phi_{1} \phi_{2} \phi_{3} \phi_{4}:$, we obtain

$$
\begin{aligned}
\partial_{\nu}^{x} & N_{2 / 1}^{v}(x, y)+[x \leftrightarrow y] \\
& =C^{v} \partial_{\nu}^{x}(\delta(x-y) M(x))+[x \leftrightarrow y] \\
& =C^{v} \delta(x-y) \partial_{v} M(x) .
\end{aligned}
$$

In addition, $\left.\partial_{v}^{x} T_{2 / 1}^{v}\right|_{\text {tree }} ^{0}(x, y)+[x \leftrightarrow y]$ also contains some $(\partial) \delta$-terms, generated due to the propagator equation $\left(\square+m^{2}\right) \Delta_{m}^{F}=\delta$ :

3. If $P^{v}=b: \partial^{v} \phi F:+\cdots$ and $L_{1}=a: \phi E:+\cdots$, then the contraction of $\partial^{v} \phi(x)$ with $\phi(y)$ gives a propagator $-i \partial^{v} \Delta_{m}^{F}(x-y)$, and on computing its divergence we find the contribution

$$
\begin{aligned}
\partial_{\nu}^{x} & \left.T_{2 / 1}^{v}\right|_{\text {tree }} ^{0}(x, y)+[x \leftrightarrow y] \\
& =-2 i b a \delta(x-y): F(x) E(x):+\cdots .
\end{aligned}
$$

4. With $P^{v}$ as before and $L_{1}(y)=a_{\mu}: \partial^{\mu} \phi(y) E(y):+\cdots$ the contraction of $\partial^{v} \phi(x)$ with $\partial^{\mu} \phi(y)$ gives a propagator $i \partial^{\nu} \partial^{\mu} \Delta_{m}^{F}(x-y)$. On computing the divergence we now obtain a $\partial \delta$-term, which we transform into a $\delta$-term by using the following identity

$$
M(y) \partial_{\nu}^{x} \delta(x-y)=M(x) \partial_{\nu}^{x} \delta(x-y)+\partial_{\nu} M(x) \delta(x-y) ;
$$

so that

$$
\begin{aligned}
\left.\partial_{\nu}^{x} T_{2 / 1}^{v}\right|_{\text {tree }} ^{0}(x, y)+[x \leftrightarrow y] \\
=i b a_{\mu} \partial^{\mu} \delta(x-y): F(x) E(y):+[x \leftrightarrow y]+\cdots \\
=i b a_{\mu} \delta(x-y)\left(: F(x) \partial^{\mu} E(x):-: \partial^{\mu} F(x) E(x):\right) \\
\quad+\cdots .
\end{aligned}
$$

After the transformations (9) and (10), all terms remaining in (8) are of the form $c_{1} \delta(x-y): \partial \phi_{1} \phi_{2} \phi_{3} \phi_{4}(x)$ : or $c_{2} \delta(x-y): \phi_{1} \phi_{2} \phi_{3} \phi_{4}(x)$ :. These are linearly independent. Therefore (8) is equivalent to a system of $c$-number equations, obtained by equating the coefficients belonging to the same Wick monomial.

\subsection{The second CGI method}

Basic model-building according to CGI uses tree-diagram calculations. In this connection, and alternatively to the previous method, models satisfying CGI at tree level can also be obtained by using that classical BRST invariance of the Lagrangian implies CGI for tree diagrams to all orders [45]. The ideas behind this can be summarized thus: given a BRST-invariant free theory, that is

$s_{0} L_{0}=\partial_{\mu} I_{0}^{\mu}=: \partial \cdot I_{0} \quad$ for some local $I_{0}$,

with $L_{0}$ quadratic in the fields, seek deformations $L_{0} \rightarrow$ $L_{\text {tot }}=\sum_{n=0}^{\infty} \kappa^{n} L_{n}$ and $s_{0} \rightarrow s=\sum_{n=0}^{\infty} \kappa^{n} s_{n}$ (with $L_{n}, s_{n}$ satisfying some obvious properties), such that

$s L_{\mathrm{tot}}=\partial \cdot I \quad$ where $I^{\mu}=\sum_{n=0}^{\infty} \kappa^{n} I_{n}^{\mu}$

is some local power series. Here $L_{\text {tot }}$ is assumed to contain only first-order derivatives; have a look at [27]. BRST invariance of the Lagrangian in this sense implies CGI for tree diagrams to all orders by the following: in the case of a constant coupling $\kappa$ formula (11) implies conservation of the corresponding classical Noether (BRST) current: $\partial \cdot j_{\kappa, \text { class }}=0$. Replacing $\kappa$ by $\kappa g$, for a test function $g$, a generalized current conservation can be derived from (11):

$\partial \cdot j_{\kappa g, \text { class }}(x)=\partial g(x) \cdot P_{\kappa g, \text { class }}(x)$,

where $P_{\kappa g \text {,class }}(x)$ is that classical interacting vector field which agrees for $\kappa=0$ with the $Q$-vertex $P$ of (5), more precisely with the corresponding classical (local) field polynomial. Since the classical limit of an interacting perturbative quantum field is given by the contribution of connected tree diagrams, current conservation (12) can be expressed as a tree-diagram relation in perturbative quantum field theory. Smearing out this relation with suitable test functions, conservation of the BRST current goes over to commutation with the free BRST charge $Q_{\text {in }}$ belonging to the conserved Noether current of the symmetry $s_{0} L_{0}=\partial \cdot I_{0}$. In this way the CGI relation (7) for tree diagrams to all orders is obtained.

For theories with MVB one can use that, generically, models coming from SSB are classically BRST invariant in the sense of (11); this will be spelled out in Sect. 5.4. Therefore they satisfy CGI at tree level. Most likely, the two methods outlined here are equivalent, in the sense that the sets of 
allowed models are the same. It remains that, whereas the first method amounts to a direct (perchance tedious) search for the general solution of the CGI conditions for tree diagrams, we do not know whether the second yields the most general solution as well.

\section{Mass and interaction patterns}

Consider a model with $t$ intermediate vector bosons $A_{a}$ in all, of which any may be in principle massive or massless. Let there be $r$ massive ones $(a=1, \ldots, r$, with masses $\left.m_{a}>0\right)$ and $s$ massless $(a=r+1, \ldots, r+s)$, so $t=r+s$. They are accompanied by $z$ physical scalar particles $\varphi_{p}$ of respective masses $\mu_{p}$. The free BRST transformation $s_{0} \equiv\left[Q_{\mathrm{in}}, \cdot\right]_{\mp}$ is a superderivation commuting with partial derivatives, hence given by its action on the basic fields:

$$
\begin{aligned}
& s_{0} A_{a}^{\mu}=\partial^{\mu} u_{a}, \quad s_{0} B_{a}=m_{a} u_{a}, \quad s_{0} u_{a}=0, \\
& s_{0} \tilde{u}_{a}=-\left(\partial \cdot A_{a}+m_{a} B_{a}\right), \quad s_{0} \varphi_{p}=0 .
\end{aligned}
$$

Here we let $B_{a}$ denote the Stückelberg field associated to the vector field $A_{a}$; in case $A_{a}$ is massless, $B_{a}$ drops out. The total bosonic interaction Lagrangian is of the form $L_{\text {int }}=\kappa L_{1}+\kappa^{2} L_{2}$. For $L_{1}$ make the following Ansatz (with unknown coefficients $f_{* * *}^{*}$ in the terms below). Let $L_{1}=$ $L_{1 B}+L_{1 \varphi}$, the higgs-free cubic couplings being $L_{1 B}=$ $L_{1}^{1}+L_{1}^{2}+L_{1}^{3}+L_{1}^{4}$ and $L_{1 \varphi}=L_{1}^{5}+L_{1}^{6}+L_{1}^{7}+L_{1}^{8}+L_{1}^{9}+$ $L_{1}^{10}+L_{1}^{11}$ being the couplings involving physical scalars. In the Feynman gauge, the allowed higgs-free cubic couplings are

$$
\begin{aligned}
L_{1}^{1} & =f_{a b c}\left[A_{a} \cdot\left(A_{b} \cdot \partial\right) A_{c}-u_{b}\left(A_{a} \cdot \partial \tilde{u}_{c}\right)\right] \\
L_{1}^{2} & =f_{a b c}^{2}\left(A_{a} \cdot A_{b}\right) B_{c} \\
L_{1}^{3} & =f_{a b c}^{3}\left[\left(A_{a} \cdot \partial B_{c}\right) B_{b}-\left(A_{a} \cdot \partial B_{b}\right) B_{c}\right] \\
L_{1}^{4} & =f_{a b c}^{4} \tilde{u}_{a} u_{b} B_{c}
\end{aligned}
$$

and the remaining ones, involving higgses, are

$$
\begin{aligned}
& L_{1}^{5}=f_{a b p}^{5}\left[\left(A_{a} \cdot \partial \varphi_{p}\right) B_{b}-\left(A_{a} \cdot \partial B_{b}\right) \varphi_{p}\right] ; \\
& L_{1}^{6}=f_{a q p}^{6}\left[\left(A_{a} \cdot \partial \varphi_{p}\right) \varphi_{q}-\left(A_{a} \cdot \partial \varphi_{q}\right) \varphi_{p}\right] ; \\
& L_{1}^{7}=f_{a b p}^{7}\left(A_{a} \cdot A_{b}\right) \varphi_{p} ; \\
& L_{1}^{8}=f_{a b p}^{8} \tilde{u}_{a} u_{b} \varphi_{p} ; \\
& L_{1}^{9}=f_{a b p}^{9} B_{a} B_{b} \varphi_{p} ; \\
& L_{1}^{10}=f_{a p q}^{10} B_{a} \varphi_{p} \varphi_{q} ; \\
& L_{1}^{11}=f_{p q r}^{11} \varphi_{p} \varphi_{q} \varphi_{r} .
\end{aligned}
$$

As products of field operators, these monomials are understood to be normally ordered. Some symmetry relations of the coefficients under exchange of indices are evident from the definition. Because the dimension of the Lagrangian must be $M^{4}$ in natural units, and the boson field dimension is 1 in our formulation, the coefficients $f, f^{3}, f^{5}, f^{6}$ are dimensionless, and $f^{2}, f^{4}, f^{7}, \ldots, f^{11}$ have dimension of mass. It is taken into account that CGI holds a term in $B_{a} B_{b} B_{c}$ to vanish. With that, the formulas (13) and (14) give the most general trilinear and renormalizable Ansatz modulo divergence terms and $s_{0}$-coboundaries.

We list the determination of the couplings in terms of the $f_{a b c}$ and the pattern of masses imposed by CGI at orders $\kappa$ and $\kappa^{2}$, still essentially in the version of [35].

1. As repeatedly indicated, and like in Sect. 1.2, with independence of the masses CGI unambiguously leads to gauge fields with real coupling parameters $f_{a b c}$ that are totally antisymmetric and satisfy the Jacobi identity: that is, to generalized Yang-Mills theories on reductive Lie algebras. This is remarkable.

2. When all $A_{a}$ are massless, there is no need to add physical or unphysical scalars for renormalizability, and only $L_{1}^{1}$ (and later, the quartic coupling $L_{2}^{1}$ ) survive. They of course coincide respectively with the first- and secondorder part of the usual Yang-Mills Lagrangian. In particular: CGI gives rise to gluodynamics.

3. The relation

$2 m_{c} f_{a b c}^{2}=\left(m_{b}^{2}-m_{a}^{2}\right) f_{a b c}$

holds. Thus if $m_{c}=0$ and $f_{a b c} \neq 0$, then $m_{a}=m_{b}$ necessarily. And if $m_{c} \neq 0$, then

$f_{a b c}^{2}=f_{a b c} \frac{m_{b}^{2}-m_{a}^{2}}{2 m_{c}}$.

The useful relation between masses and structure constants:

$$
\left(m_{b}^{2}-m_{a}^{2}\right) \sum_{c: m_{c}=0}\left(f_{a b c}\right)^{2}=0
$$

follows directly from (15).

4. The relation

$2\left(f_{b c a}^{3} m_{a}-f_{a c b}^{3} m_{b}\right)=f_{a b c} m_{c}$

holds. From this, after multiplication by $m_{c}$ and cyclic permutation, one obtains the important formula

$f_{a b c}^{3}=f_{a b c}\left(m_{b}^{2}+m_{c}^{2}-m_{a}^{2}\right) / 4 m_{b} m_{c}$.

If either $m_{b}$ or $m_{c}$ vanishes, then $f_{a b c}^{3}=0$. 
5. $f_{a b c}^{4}=f_{a b c}\left(m_{c}^{2}-m_{b}^{2}+m_{a}^{2}\right) / 2 m_{c}$.

6. In the non-Abelian case, when some $A_{a}$ are massive, coefficients $f^{5}$ to $f^{11}$ cannot all vanish: renormalizability asks for physical Higgs bosons. The $L_{1}^{5}$ and $L_{1}^{6}$ terms are the nub of the problem. Reference [35] claims that $L_{1}^{6}$ is just zero and that the coefficients of $L_{1}^{5}$ are diagonal in the sense that $f_{a b p}^{5}=C_{5 p} m_{a} \delta_{a b}$, where the $C_{5 p}$ (with dimension $M^{-1}$ ) are independent of $a$; but this is only warranted when there is a single higgs field, for which the $L_{1}^{6}$ term is absent. A relatively involved expression, given in the next subsection, ties this key coupling with the structure constants and the masses.

7. $f_{a b p}^{7}=-f_{a b p}^{8}=m_{b} f_{a b p}^{5}$. This is $C_{5} m_{a}^{2} \delta_{a b}$ when $z=1$.

8. Because $f^{7}$ is obviously symmetric in the first two indices, so too is $f^{8}$. Now, the symmetry for $f^{8}$ implies

$m_{b} f_{a b p}^{5}=m_{a} f_{b a p}^{5}$.

Note that $f_{a b p}^{5}=0$ if $m_{a}=0$ or $m_{b}=0$ : for $m_{b}=0$ this is clear (no Stückelberg field $B_{b}$ ), and for $m_{a}=0$ it follows from (19).

9. $f_{a b p}^{9}=-\left(\mu_{p}^{2} / 2 m_{a}\right) f_{a b p}^{5}$ for $m_{a}>0 ; f_{a b p}^{9}=0$ if $m_{a}=0$. This is $-\frac{1}{2} C_{5} m_{H}^{2} \delta_{a b}$ when $z=1$, with $\mu_{1} \equiv m_{H}$.

10. $f_{a p q}^{10}=\frac{\mu_{q}^{2}-\mu_{p}^{2}}{m_{a}} f_{a p q}^{6}$ for $m_{a}>0$, with $f_{a p q}^{10}=0$ if $m_{a}=$ 0 . This vanishes when $z=1$.

11. The $f_{p q r}^{11}$ are not determined, except (with the help of third-order tree graphs) in the case of only one higgs; then $f^{11}=-\frac{1}{2} C_{5} m_{H}^{2}$.

\subsection{The first CGI parameter constraint}

In the preceding subsection we have listed all conditions coming from CGI for first and second-order tree diagrams which determine directly the coupling parameters $f_{* * *}^{*}$ in terms of $f_{a b c}$ and the masses. However, for second-order tree diagrams CGI gives further constraints relating the couplings and the MVB masses. Using implicit summation on repeated indices, the first of those is

$$
\begin{aligned}
& f_{a j p}^{5} f_{d b p}^{5}-f_{a b p}^{5} f_{d j p}^{5} \\
& =\frac{m_{j}^{2}+m_{b}^{2}-m_{c}^{2}}{2 m_{j} m_{b}} f_{d a c} f_{c b j} \\
& \quad+\left(\frac{m_{k}^{2}+m_{j}^{2}-m_{d}^{2}}{m_{j} m_{k}} \frac{m_{k}^{2}+m_{b}^{2}-m_{a}^{2}}{4 m_{b} m_{k}} f_{d j k} f_{a b k}\right. \\
& \quad-[a \leftrightarrow d]),
\end{aligned}
$$

if $m_{b}, m_{j}>0$. The sum over $c$ is over all gauge bosons and the sum over $k$ runs only over massive ones.
In particular, setting $j=a$ and $d=b \neq a$, one infers that

$$
\begin{aligned}
f_{\text {aap }}^{5} f_{b b p}^{5}-f_{a b p}^{5} f_{\text {bap }}^{5} & \\
= & \frac{1}{2 m_{a} m_{b}}\left[\sum_{c=1}^{t}\left(m_{a}^{2}+m_{b}^{2}-m_{c}^{2}\right)\left(f_{a b c}\right)^{2}\right. \\
& \left.\quad+\sum_{k: m_{k} \neq 0} \frac{\left(m_{a}^{2}-m_{b}^{2}\right)^{2}-m_{k}^{4}}{2 m_{k}^{2}}\left(f_{a b k}\right)^{2}\right] .
\end{aligned}
$$

On the one hand this relation allows us to compute $f^{5}$ from the masses and the structure constants; on the other hand, since it is valid for any $b \neq a$, it implies direct relations between the masses and the structure constants. ${ }^{5}$

It will help to reorganize (21), separating the massless from the massive bosons in the sum. If $m_{k} \neq 0$, the coefficient of $\left(f_{a b k}\right)^{2} / 4 m_{a} m_{b} m_{k}^{2}$ is

$$
\begin{gathered}
2 m_{k}^{2}\left(m_{a}^{2}+m_{b}^{2}-m_{k}^{2}\right)+\left(m_{a}^{2}-m_{b}^{2}\right)^{2}-m_{k}^{4} \\
=\left(m_{a}^{2}+m_{b}^{2}+m_{k}^{2}\right)^{2}-4\left(m_{a}^{2} m_{b}^{2}+m_{k}^{4}\right) .
\end{gathered}
$$

Thus the main consequence of (20) can be written, for $a \neq b$ with $m_{b} \neq 0$, as:

$$
\begin{aligned}
& 4 m_{a} m_{b} \sum_{p=1}^{z}\left|\begin{array}{cc}
f_{a a p}^{5} & f_{a b p}^{5} \\
f_{b a p}^{5} & f_{b b p}^{5}
\end{array}\right| \\
& =2\left(m_{a}^{2}+m_{b}^{2}\right) \sum_{d: m_{d}=0}\left(f_{a b d}\right)^{2} \\
& \quad+\sum_{k: m_{k} \neq 0} \frac{\left(f_{a b k}\right)^{2}}{m_{k}^{2}}\left[\left(m_{a}^{2}+m_{b}^{2}+m_{k}^{2}\right)^{2}\right. \\
& \left.-4\left(m_{a}^{2} m_{b}^{2}+m_{k}^{4}\right)\right] .
\end{aligned}
$$

This first constraint and (15), with their respective consequences (22) and (16), restrict strongly the masses of the gauge bosons (Sects. 5.2-5.3).

\section{The relation between CGI and SSB}

The primary aim of this section is to work out explicitly the connection of model building by CGI to the SSB approach. In particular we show that one obtains the covariant derivative of the scalar fields, that is, the "minimal coupling" recipe. A related aim is to disprove the claim [46] about standard GUT models not satisfying CGI at tree level, that would contradict our aforementioned statement. Finally, we collect information on the $L_{2}$ piece of the Lagrangian. We restate that:

${ }^{5}$ We observe that Scharf writes the previous equation differently, since he mistakenly "derived" $f_{a b p}^{5}=0$ when $a \neq b$. 
- From CGI for spin-one particles, one is led to discover the gauge symmetry: the coupling parameters $f_{a b c}$ in $L_{1}^{1}$ are the structure constants of a reductive Lie algebra, and the other couplings $f_{* * *}^{*}$ in $L_{1}^{*}$ are determined by the $f_{a b c}$ and the masses. Knowledge of this hidden symmetry is of course very useful, but not needed a priori within CGI.

- In the opposite direction, i.e. postulating the underlying gauge symmetry, we expect that models built by SSB be classically BRST invariant, and hence satisfy CGI at tree level to all orders [45].

\subsection{Reinterpreting the first constraint from CGI}

Using (18), the main obstruction (20) is rewritten

$$
\begin{aligned}
f_{a j p}^{5} f_{d b p}^{5}-f_{a b p}^{5} f_{d j p}^{5}= & 2 f_{d a c} f_{c b j}^{3}+4 f_{a j k}^{3} f_{d k b}^{3} \\
& -4 f_{a b k}^{3} f_{d k j}^{3} .
\end{aligned}
$$

In view of (13) and (14) it is clear that $f^{3}$ and $f^{5}$ should be related. We introduce the notation

$$
\left(F^{a}\right)_{b c}=-2 f_{a b c}^{3}
$$

for $r \times r$ skewsymmetric matrices $F^{a}$. If we provisionally assume that only one physical scalar is present $(z=1)$, let $G^{a}$ be $r \times 1$ matrices (there are $r+s$ of these) given by

$\left(G^{a}\right)_{j}=-f_{a j}^{5}$,

and form the $(r+1) \times(r+1)$ skewsymmetric matrices, for $a=1, \ldots, r+s$ :

$S^{a}=\left(\begin{array}{cc}F^{a} & G^{a} \\ -{ }^{t} G^{a} & 0\end{array}\right)$,

with ${ }^{t} G^{a}$ being the transpose of $G^{a}$.

Relation (23) corresponds to the left upper corner of the commutator bracket

$$
\left[S^{a}, S^{d}\right]=f_{a d c} S^{c} .
$$

Employing $f_{a b}^{5}=C_{5} m_{a} \delta_{a b}$ and (17), one sees that the other corners of this bracket formula are fulfilled, too. Thus (23) means that $f^{3}, f^{5}$ taken together define a real skewsymmetric matrix representation of the gauge group with dimensionless entries, for only one higgs.

\subsection{How the covariant derivative arises from CGI}

When more than one higgs is present, one should admit terms like $\left(A_{a} \cdot \partial \varphi_{q}\right) \varphi_{p}-\left(A_{a} \cdot \partial \varphi_{p}\right) \varphi_{q}$, and so we have done in (14). In this case a second constraint is found,

$$
\begin{aligned}
0= & f_{a b c} f_{d c p}^{8}-f_{d b k}^{4} f_{a k p}^{5}+f_{d a k}^{4} f_{b k p}^{5}+2 f_{a p v}^{6} f_{d b v}^{8} \\
& -2 f_{b p v}^{6} f_{d a v}^{8} .
\end{aligned}
$$

Here $a, b, d, p$ are fixed; the summation indices are $c=$ $1, \ldots, r+s ; k=1, \ldots, r ;$ and $v=1, \ldots, z$. The right hand side is the coefficient of the term $\left[u_{a} u_{b} \tilde{u}_{d} \varphi_{p}\right](x) \delta(x-y)$ on the right hand side of the CGI condition (8) for $n=2$. We point out that the expression must be antisymmetric in $a \leftrightarrow b$ because $u_{a} u_{b} \tilde{u}_{d} \varphi_{p}$ is. There is no contribution coming from $\left[Q_{\text {in }}, N_{2}\right]$-hence the zero on the left hand sidesince quartic terms involving ghost fields $u, \tilde{u}$ are not admitted here. This can be justified by the second CGI method.

We finally line up the following system of constraints:

$$
\begin{aligned}
& 4 f_{a d k}^{3} f_{b k e}^{3}-4 f_{b d k}^{3} f_{a k e}^{3}-f_{a d v}^{5} f_{b e v}^{5}+f_{b d v}^{5} f_{a e v}^{5} \\
& =-2 f_{a b c} f_{c d e}^{3}, \quad\left(m_{d}>0, m_{e}>0\right) \\
& 2 f_{a d k}^{3} f_{b k p}^{5}-2 f_{b d k}^{3} f_{a k p}^{5}-2 f_{a d v}^{5} f_{b p v}^{6}+2 f_{b d v}^{5} f_{a p v}^{6} \\
& =-f_{a b c} f_{c d p}^{5}, \quad\left(m_{d}>0\right) \\
& -f_{a k p}^{5} f_{b k q}^{5}+f_{b k p}^{5} f_{a k q}^{5}+4 f_{a p v}^{6} f_{b v q}^{6}-4 f_{b p v}^{6} f_{a v q}^{6} \\
& =-2 f_{a b c} f_{c p q}^{6} .
\end{aligned}
$$

The first equation is the by now familiar basic constraint of Sect. 3.1; the second is the previously displayed equation divided by $-m_{d}$. The derivation of these constraints from CGI is discussed in Appendix B.

Let us reintroduce the matrices $\left(G^{a}\right)_{d p}=-f_{a d p}^{5}$, which are now $r \times z$, and introduce the $z \times z$ ones:

$\left(H^{a}\right)_{p q}=-2 f_{a p q}^{6}$.

Then the system (25) amounts to the triplet of matrix equations,

$\left[F^{a}, F^{b}\right]-G^{a t} G^{b}+G^{b t} G^{a}=f_{a b c} F^{c}, \quad(r \times r)$

$F^{a} G^{b}-F^{b} G^{a}+G^{a} H^{b}-G^{b} H^{a}=f_{a b c} G^{c}, \quad(r \times z)$

$-{ }^{t} G^{a} G^{b}+{ }^{t} G^{b} G^{a}+\left[H^{a}, H^{b}\right]=f_{a b c} H^{c} . \quad(z \times z)$.

Putting it all together in the skewsymmetric $(r+z) \times(r+z)$ package

$S^{a}=\left(\begin{array}{cc}F^{a} & G^{a} \\ -{ }^{t} G^{a} & H^{a}\end{array}\right)=\left(\begin{array}{cc}-2 f_{a * *}^{3} & -f_{a * \star}^{5} \\ f_{a \star *}^{5} & -2 f_{a \star \star}^{6}\end{array}\right)$,

this generalizes the gauge-group representation $\left[S^{a}, S^{b}\right]=$ $f_{a b c} S^{c}$ mooted in (24). In fine, the key couplings $f^{5}, f^{6}$ of the physical scalars are constrained by this algebraic relation in terms of the "known" $f^{3}$ couplings. ${ }^{6}$

\footnotetext{
${ }^{6}$ Also within CGI, in an analogous way the coupling of vector bosons to fermions induces a gauge-group representation among the latter fields.
} 
Moreover, we contend that the representation above is the one yielding the covariant derivative on the scalar multiplets of the "minimal coupling" recipe, written in real form. More explicitly, let $\eta$ be a scalar multiplet assembled from the Stückelberg fields and the higgses by

$\eta^{t}:=\left(B_{1}, \ldots, B_{r}, \varphi_{1}+v_{1}, \ldots, \varphi_{z}+v_{z}\right)$,

where the field shifts $v_{p}$ are real numbers. We make two assertions. The first is a statement about the corresponding hidden gauge symmetry; namely that the multiplet $\eta$ transforms with the representation $S^{a}=: S\left(T_{a}\right)$, with $T_{a}$ the generators of the gauge Lie algebra, and hence

$D^{\mu} \eta:=\left(\partial^{\mu}+\kappa A_{a}^{\mu} S^{a}\right) \eta$,

is the covariant derivative of $\eta$. Our second claim is that the minimal coupling recipe holds true, in the sense that, with a suitable choice of the $v_{p}$,

$$
\begin{gathered}
\frac{1}{2} \partial \eta^{t} \cdot \partial \eta+\frac{\kappa}{2}\left(\left(A_{a} \cdot \partial \eta^{t}\right) S^{a} \eta-\eta^{t} S^{a}\left(A_{a} \cdot \partial \eta\right)\right) \\
-\frac{\kappa^{2}}{4}\left(A_{a} \cdot A_{b}\right) \eta^{t}\left[S_{a}, S_{b}\right]_{+} \eta
\end{gathered}
$$

agrees with what one obtains by the CGI method for the scalar-gauge Lagrangian, that is, besides the kinetic terms of the $B$ - and $\varphi$-fields, the vector-boson mass term, plus an $(A \cdot \partial B)$ term, plus the trilinear couplings $L_{1}^{2}+L_{1}^{3}+L_{1}^{5}+$ $L_{1}^{6}+L_{1}^{7}$ of the gauge fields $A$ to the scalars $(B, \varphi)$ considered in (13) and (14), plus the quartic terms $L_{2}^{2}+L_{2}^{3}+L_{2}^{4}$ defined in Sect. 4.3 right below. (Strictly speaking, within CGI the shift of the fields by the $v_{p}$ is not required at second order. However, it is convenient for our purposes. In examples, the "correct" choice of $v_{p}$ can be obtained from a comparison with SSB: the fields $\varphi_{p}+v_{p}$ are the ones of the "unbroken" model with its full gauge symmetry.) We routinely verify our assertions in the example models constructed by CGI in the next section. Therefore our procedure derives within CGI the crucial piece $\frac{1}{2}(D \eta)^{t} \cdot D \eta$ of the Lagrangian. Indeed this provides the crowning point of the construction.

\subsection{On the quartic couplings}

There are quartic terms

$L_{2}=\frac{1}{2}\left(L_{2}^{1}+L_{2}^{2}+L_{2}^{3}+L_{2}^{4}+L_{2}^{5}+L_{2}^{6}+L_{2}^{7}\right)$, with obvious symmetries as before, of the following form:

$L_{2}^{1}=h_{b c d e}^{1}\left(A_{b} \cdot A_{d}\right)\left(A_{c} \cdot A_{e}\right)$,

$L_{2}^{2}=h_{a b c d}^{2}\left(A_{a} \cdot A_{b}\right) B_{c} B_{d}$,

$L_{2}^{3}=h_{a b c p}^{3}\left(A_{a} \cdot A_{b}\right) B_{c} \varphi_{p}$,

$L_{2}^{4}=h_{a b p q}^{4}\left(A_{a} \cdot A_{b}\right) \varphi_{p} \varphi_{q}$,

$L_{2}^{5}=h_{a b c d}^{5} B_{a} B_{b} B_{c} B_{d}$,

$L_{2}^{6}=h_{a b p q}^{6} B_{a} B_{b} \varphi_{p} \varphi_{q}$,

$L_{2}^{7}=h_{p q r s}^{7} \varphi_{p} \varphi_{q} \varphi_{r} \varphi_{s}$.

A complete account of the permitted quartic terms would take us too far afield. For instance, to answer the question of whether models are completely fixed in the general case by CGI and by requiring that the number of higgs fields be as small as possible, one needs a complete study of the treelevel third-order conditions, as well as to revisit some corners of the second-order conditions, here unexplored. This is better left for another paper. We limit ourselves to reporting on what can be gleaned from the foregoing and calculations analogous to the ones performed in the coming Sect. 5 and in Appendix B.

One finds from CGI $h_{b c d e}^{1}=-\frac{1}{2} f_{a b c} f_{a d e}$ as thoroughly expected: it just yields the quartic part in the Yang-Mills Lagrangian, irrespectively of masses.

Now, it is plain what $L_{2}^{2}, L_{2}^{3}, L_{2}^{4}$ of formula (28) must be. Have a look back at (27). According to our results on minimal coupling from CGI at second order, these terms in the interaction Lagrangian are generated by suitable combinations not involving $v$ in $-\frac{1}{4}\left(A_{a} \cdot A_{b}\right) \eta^{t}\left[S_{a}, S_{b}\right]_{+} \eta$. Therefore, taking into account the factor $\frac{1}{2}$ in the definitions, one finds:

- For the higgs-free term $L_{2}^{2}, h_{a b c d}^{2}=-2 f_{a c k}^{3} f_{b k d}^{3}-$ $2 f_{b c k}^{3} f_{a k d}^{3}+\frac{1}{2} f_{a c v}^{5} f_{b d v}^{5}+\frac{1}{2} f_{b c v}^{5} f_{a d v}^{5}$. Here and in the subsequent formulas we sum over repeated indices. This is symmetric under $a \leftrightarrow b$ and $c \leftrightarrow d$, as it should be.

$-h_{a b c p}^{3}=-2 f_{a c k}^{3} f_{b k p}^{5}-2 f_{b c k}^{3} f_{a k p}^{5}+2 f_{a c v}^{5} f_{b p v}^{6}+$ $2 f_{b c v}^{5} f_{a p v}^{6}$. This is symmetric in $a, b$.

$-h_{a b p q}^{4}=-2 f_{a p v}^{6} f_{b v q}^{6}-2 f_{b p v}^{6} f_{a v q}^{6}+\frac{1}{2} f_{a k p}^{5} f_{b k q}^{5}+$ $\frac{1}{2} f_{b k p}^{5} f_{a k q}^{5}$. This is symmetric under $a \leftrightarrow b$ and $p \leftrightarrow q$.

For the higgs-free term $L_{2}^{5}$ we find, for $a, b, c, d \leq r$ :

$h_{a b c d}^{5}=-\frac{\mu_{p}^{2}}{12}\left(\frac{f_{a b p}^{5} f_{c d p}^{5}}{m_{a} m_{c}}+\frac{f_{a c p}^{5} f_{b d p}^{5}}{m_{a} m_{b}}+\frac{f_{a d p}^{5} f_{c b p}^{5}}{m_{a} m_{c}}\right)$.

This ought to be symmetric under exchanges of $a, b, c, d$, and indeed it is: the relations $m_{b} f_{a b p}^{5}=m_{a} f_{b a p}^{5}$ save the 
day. For $L_{2}^{6}$ we find:

$$
\begin{aligned}
h_{a b p q}^{6}= & \frac{\mu_{p}^{2}+\mu_{q}^{2}}{4 m_{a} m_{b}}\left(f_{a c p}^{5} f_{b c q}^{5}+f_{a c q}^{5} f_{b c p}^{5}\right)+\frac{3}{m_{a}} f_{p q u}^{11} f_{a b u}^{5} \\
& +\frac{2 \mu_{u}^{2}-\mu_{q}^{2}-\mu_{p}^{2}}{m_{a} m_{b}}\left(f_{\text {aup }}^{6} f_{b u q}^{6}+f_{\text {auq }}^{6} f_{b u p}^{6}\right) \\
& +\frac{\left(m_{a}^{2}-m_{b}^{2}\right)\left(\mu_{p}^{2}-\mu_{q}^{2}\right)}{2 m_{a} m_{b} m_{k}^{2}} f_{a b k} f_{k p q}^{6} .
\end{aligned}
$$

This has the required symmetries under $a \leftrightarrow b, p \leftrightarrow q$; it is undetermined at second order, because $f^{11}$ is.

Finally, $h^{7}$ is undetermined at second order. CGI for third-order tree diagrams yields conditions restricting $h^{7}$ and $f^{11}$ (via conditions on $h^{6}$ ), which in the case $z=1$ determine these parameters uniquely; see the next subsection. The procedure was explained in [42, Sect. 5], with calculations given in detail for the SM; consult [35] as well.

\subsection{Quartic couplings for models with only one higgs}

For the case $z=1$, with the $\varphi$-index suppressed, we obtain:

$$
\begin{aligned}
\text { 1. } & h_{a b c d}^{2}=\sum_{k: m_{k} \neq 0} \frac{1}{8 m_{c} m_{d} m_{k}^{2}}\left(f_{a c k} f_{b d k}\left(m_{c}^{2}+m_{k}^{2}-m_{a}^{2}\right) \times\right. \\
& \left.\left(m_{d}^{2}+m_{k}^{2}-m_{b}^{2}\right)+[a \leftrightarrow b]\right)+\frac{1}{2} C_{5}^{2} m_{a} m_{b}\left(\delta_{a c} \delta_{b d}+\right. \\
& \left.\delta_{a d} \delta_{b c}\right) .
\end{aligned}
$$

2. $h_{a b c}^{3}=2 f_{a b c}^{2} C_{5}=f_{a b c} C_{5}\left(m_{b}^{2}-m_{a}^{2}\right) / m_{c}$.

3. $h_{a b}^{4}=C_{5}^{2} m_{a}^{2} \delta_{a b}$.

4. $h_{a b c d}^{5}=\frac{1}{3}\left(\delta_{a b} \delta_{c d}+\delta_{a c} \delta_{b d}+\delta_{a d} \delta_{b c}\right) h^{7} ; h_{a b}^{6}=2 \delta_{a b} h^{7}$; $h_{7}=-\frac{1}{4} C_{5}^{2} m_{H}^{2}$, independently of indices $\leq r$.

This allows us to peek at the purely scalar sector with one higgs. Including its mass term, it becomes

$$
\begin{aligned}
- & \frac{1}{2} m_{H}^{2} \varphi^{2}+\kappa\left(f_{a b}^{9} B_{a} B_{b} \varphi+f^{11} \varphi^{3}\right) \\
& +\frac{\kappa^{2}}{2}\left(h_{a b c d}^{5} B_{a} B_{b} B_{c} B_{d}+h_{a b}^{6} B_{a} B_{b} \varphi^{2}+h^{7} \varphi^{4}\right) \\
= & -\frac{m_{H}^{2}}{2}\left(\varphi^{2}+\kappa C_{5}\left(\sum_{a=1}^{r} B_{a}^{2}+\varphi^{2}\right) \varphi\right. \\
& \left.+\frac{\kappa^{2}}{4} C_{5}^{2}\left(\sum_{a=1}^{r} B_{a}^{2}+\varphi^{2}\right)^{2}\right) \\
= & -\frac{\kappa^{2} m_{H}^{2} C_{5}^{2}}{8}\left(\frac{2 \varphi}{\kappa C_{5}}+\varphi^{2}+|\boldsymbol{B}|^{2}\right)^{2}=:-V(\varphi, \boldsymbol{B}) .
\end{aligned}
$$

These formulas are correctly given in [35]. The potential exhibits a characteristic $O(r+1)$ symmetry [64]. Leaving aside the Stückelberg fields, it has a minimum at $\varphi=0$.
Hence, the physical higgs field can be realized in an ordinary Fock representation, with a unique vacuum and vanishing vacuum expectation value.

\section{The CGI methods in practice}

The plan of this section is as follows. We first attack from the perspective of the first CGI approach the simplest example one can think of - dealt with only summarily in [35]. We investigate next models with several massive vector bosons, but one physical higgs $(z=1)$ only, using the first CGI method as in Sect. 3.1. One may derive here the possible mass patterns of the gauge bosons by taking only the consequences of (15) and (20) into account. Of course, to show that the resulting models indeed satisfy CGI at tree level, one must verify all $c$-number identities expressing (5) and (7) on that level. The solutions of those equations that we work out are compatible with the CGI conditions at all orders. We finally look at causal gauge invariance for models with scalar fields in the adjoint. All along, we flesh out the relation between CGI and SSB whose theoretical underpinning was derived in the previous section.

\subsection{The toy model}

The case $r=1, s=0, z=1$ leads to an Abelian model in which all the terms $L_{1}^{5}$ to $L_{1}^{11}$ with the higgs-like field $\varphi$ appear, except $L_{1}^{6}$. All contributions of the first group, $L_{1}^{1}$ to $L_{1}^{4}$, disappear. Also $L_{2}^{1}$ and $L_{2}^{2}$ vanish. The obstructions of Sect. 3.1 play no role here. This does not sound very interesting; but it is instructive. Eleven contributions in all survive, we find that $C_{5}=1 / \mathrm{m}$ with $m$ being the mass of the spin 1 particle, and the resulting interaction Lagrangian reads

$$
\begin{aligned}
L_{\text {int }}(x)= & \kappa m(A \cdot A) \varphi-\kappa m \tilde{u} u \varphi+\kappa B(A \cdot \partial \varphi) \\
& -\kappa \varphi(A \cdot \partial B)-\frac{\kappa m_{H}^{2}}{2 m} \varphi^{3}-\frac{\kappa m_{H}^{2}}{2 m} B^{2} \varphi \\
& +\frac{\kappa^{2}}{2}(A \cdot A) \varphi^{2}+\frac{\kappa^{2}}{2}(A \cdot A) B^{2}-\frac{\kappa^{2} m_{H}^{2}}{8 m^{2}} \varphi^{4} \\
& -\frac{\kappa^{2} m_{H}^{2}}{4 m^{2}} \varphi^{2} B^{2}-\frac{\kappa^{2} m_{H}^{2}}{8 m^{2}} B^{4},
\end{aligned}
$$

where $m_{H}$ is the mass of the higgs field $\varphi$.

For the derivation of (30), recall that $T_{1}=L_{1}$ is given by the first two lines of (30). Assume that CGI to first order (5) has already been put to work, yielding the first six terms on the right hand side in (30), except that the coefficient of the $\varphi^{3}$-coupling is undetermined. The $Q$-vertex here is given by $s_{0} T_{1}=\partial \cdot P$ with $P=\kappa(m u \varphi A-u(\varphi \partial B-B \partial \varphi))$. 
Next put to work CGI for second-order tree diagrams (8). As a rule, calculations of this kind are elementary, but tedious. Unhurried readers are referred to the leisurely treatment in [65]. Also, a technically more detailed version of this paper, containing the computations pertaining here in particular, is available as hep-th/1001.0932v2.

\subsubsection{The second CGI criterion and gauge independence}

For the sake of training, we wish to verify the second CGI criterion in this example directly. So far we have adhered to the Feynman gauge, whereby the masses of gauge and Stückelberg fields coincide. To show that this restriction is not necessary, we proceed here in an arbitrary $\Lambda$-gauge $a ̀ ~ l a$ 't Hooft.

It is instructive to look first at the free model. The Stückelberg Lagrangian for a MVB is most elegantly written $[32,33]$ :

$L_{\text {Stue }}=L_{\text {kin }}(A)+\frac{m^{2}}{2}\left(A-\frac{\partial B}{m}\right)^{2}-\frac{\Lambda}{2}\left(\partial \cdot A+\frac{m}{\Lambda} B\right)^{2}$,

where $\Lambda$ is the gauge-fixing parameter. The first two terms are manifestly gauge invariant by $\delta A=\partial \alpha, \delta B=m \alpha$; however, the last one (which is the gauge-fixing term $L_{0}^{\text {gf }}$ ) is gauge invariant only if $\left(\square+m^{2} / \Lambda\right) \alpha=0$.

For the zeroth-order BRST transformation, the gaugefixing parameter $\Lambda$ appears only in $s_{0} \tilde{u}$ :

$s_{0} \tilde{u}=-(\Lambda \partial \cdot A+m B)$

and $s_{0} A=\partial u, s_{0} B=m u, s_{0} u=0, s_{0} \varphi=0$ as in the Feynman gauge. Obviously $s_{0}$ is nilpotent, except maybe for $s_{0}^{2} \tilde{u}$; we return to this point below.

The first two terms in $L_{\text {Stue }}$ are $s_{0}$-invariant, but for an unrestricted $u$-field this does not hold for $L_{0}^{\mathrm{gf}}$ :

$s_{0} L_{0}^{\mathrm{gf}}=\left(\partial \cdot s_{0} A+\frac{m}{\Lambda} s_{0} B\right) s_{0} \tilde{u}=\left(\square+\frac{m^{2}}{\Lambda}\right) u s_{0} \tilde{u}$.

For this reason one introduces a ghost Lagrangian $L_{0}^{\text {gh }}$ such that $s_{0}\left(L_{0}^{\mathrm{gf}}+L_{0}^{\mathrm{gh}}\right)$ is a divergence: with

$L_{0}^{\mathrm{gh}}=\partial \tilde{u} \cdot \partial u-\frac{m^{2}}{\Lambda} \tilde{u} u=\partial \tilde{u} \cdot s_{0} A-\frac{m}{\Lambda} \tilde{u} s_{0} B$,

we indeed obtain

$s_{0}\left(L_{0}^{\mathrm{gf}}+L_{0}^{\mathrm{gh}}\right)=\partial \cdot\left(s_{0} \tilde{u} s_{0} A\right)=: \partial \cdot I_{0}$.

Adding $L_{0}^{\mathrm{gh}}$ and the kinetic and mass terms for the higgs to $L_{\text {Stue }}$, the total free Lagrangian $L_{0}$ takes the form

$L_{0}=L_{\mathrm{kin}}(A)+\frac{m^{2}}{2}(A \cdot A)+\frac{1}{2}(\partial B \cdot \partial B)-\frac{m^{2}}{2 \Lambda} B^{2}$

$$
\begin{aligned}
& -\frac{\Lambda}{2}(\partial A)^{2}+\frac{1}{2}(\partial \varphi \cdot \partial \varphi)-\frac{m_{H}^{2}}{2} \varphi^{2}+\partial \tilde{u} \cdot \partial u \\
& -\frac{m^{2}}{\Lambda} \tilde{u} u-m \partial \cdot(A B) .
\end{aligned}
$$

It is BRST invariant in the sense that $s_{0} L_{0}=\partial \cdot I_{0}$.

Returning to nilpotence of $s_{0}$, we see that $s_{0}^{2} \tilde{u}$ vanishes modulo the free field equations:

$s_{0}^{2} \tilde{u}=-\Lambda \partial\left(s_{0} A\right)-m s_{0} B=-\Lambda \square u-m^{2} u=\Lambda \frac{\delta S_{0}}{\delta \tilde{u}}$,

where $S_{0}$ is the action corresponding to $L_{0}$. The equations of motion for the free vector field $A$ and the Stückelberg field $B$ are seen to be

$$
\left(\square+m^{2}\right) A=(1-\Lambda) \partial(\partial \cdot A) ; \quad\left(\square+\Lambda^{-1} m^{2}\right) B=0 .
$$

Thus, if any other than the Feynman gauge $\Lambda=1$ is chosen, the mass of the Stuickelberg field becomes $m / \sqrt{\Lambda}$; this is also the mass of the ghost fields $u, \tilde{u}$, and of $\partial A$.

Turning to the interacting sector, we need to verify BRST invariance for the terms (30) obtained by the first CGI method. The interacting BRST transformation $s=s_{0}+s_{1}$ has an additional term $s_{1} \sim \kappa$, given by

$s_{1} B=\kappa u \varphi, \quad s_{1} \varphi=-\kappa B u$,

and zero for the other fields. Let us look immediately at the scalar sector. In this instance $\boldsymbol{B}$ of (29) has a single component, and the point is that (32) guarantees that

$s V(\varphi, B) \propto\left(s_{0}+s_{1}\right)\left(\frac{2 m \varphi}{\kappa}+\varphi^{2}+B^{2}\right)^{2}=0$.

From $s u=0$ and $u u=0$ we obtain $s^{2} A=s^{2} B=s^{2} \varphi=0$. For $L^{\text {gh }}$ we keep the form $L^{\text {gh }}=\partial \tilde{u} \cdot s A-\frac{m}{\Lambda} \tilde{u} s B$ in (31). Note that this contributes the second term in (30), when $\Lambda=1$. Still with the new action $S$, we find that

$s^{2} \tilde{u}=-\Lambda \partial(s A)-m s B=\Lambda \frac{\delta S}{\delta \tilde{u}}$

vanishes on-shell, since only $L^{\text {gh }}$ contributes to $\delta S / \delta \tilde{u}$. The gauge-fixing part is not modified, and again

$$
\begin{aligned}
s\left(L^{\mathrm{gf}}+L^{\mathrm{gh}}\right)= & \left(\partial \cdot s A+\frac{m}{\Lambda} s B\right) s \tilde{u}+\partial(s \tilde{u}) \cdot s A \\
& -\frac{m}{\Lambda} s \tilde{u} s B \\
= & \partial \cdot(s \tilde{u} s A)=\partial \cdot I_{0} .
\end{aligned}
$$

In this particularly simple case, the vector $I$ has only components of degree zero.

We know that $s L_{\text {kin }}(A)=0$. The total Lagrangian reads

$$
L=L_{\mathrm{kin}}(A)+\frac{m^{2}}{2}\left(A-\frac{\partial B}{m}\right)^{2}+\frac{1}{2} \partial \varphi \cdot \partial \varphi-\frac{m_{H}^{2}}{2} \varphi^{2}
$$




$$
+L^{\mathrm{gf}}+L^{\mathrm{gh}}+\left(L_{\mathrm{int}}+\kappa m \tilde{u} u \varphi\right)
$$

where $L_{\mathrm{int}}$ is given by (30). It remains to verify BRST invariance of

$$
L-L_{\text {kin }}(A)-L^{\mathrm{gf}}-L^{\mathrm{gh}}+V(\varphi, B)=: L^{\eta} .
$$

As discussed in Sect. 4, these terms can be grouped into a minimal coupling recipe. As an example of the $S$-representation of that section, we have the sole $S$-matrix $\left(\begin{array}{cc}0 & -1 \\ 1 & 0\end{array}\right)$. Let us use it to the purpose. With $\eta=(B, m / \kappa+\varphi)^{t}$ and $D=$ $\partial+\kappa A S$, we obtain

$$
\begin{aligned}
& \frac{1}{2}(D \eta)^{t} \cdot D \eta \\
&= \frac{1}{2}\left(\begin{array}{c}
\partial B-\kappa(m / \kappa+\varphi) A \\
\partial \varphi+\kappa B A
\end{array}\right)^{t}\left(\begin{array}{c}
\partial B-\kappa(m / \kappa+\varphi) A \\
\partial \varphi+\kappa B A
\end{array}\right) \\
&= \frac{1}{2} \partial B \cdot \partial B+\frac{1}{2} \partial \varphi \cdot \partial \varphi+\frac{1}{2} m^{2} A \cdot A-m A \cdot \partial B \\
&+\kappa(m(A \cdot A) \varphi+B(A \cdot \partial \varphi)-\varphi(A \cdot \partial B)) \\
&+\frac{1}{2} \kappa^{2}\left((A \cdot A) \varphi^{2}+(A \cdot A) B^{2}\right)=L^{\eta},
\end{aligned}
$$

indeed providing the sought-for terms. Since the BRST variation of $\eta$ has the form of an infinitesimal gauge transformation,

$s \eta=\left(\begin{array}{c}s B \\ s \varphi\end{array}\right)=\left(\begin{array}{c}u(m+\kappa \varphi) \\ -\kappa u B\end{array}\right)=-\kappa u S \eta$,

the covariant derivative satisfies $s D \eta=-\kappa u S D \eta$, and hence $s L^{\eta}=0$. We conclude that our toy model is BRST invariant, thus causal gauge invariant on the tree level, and that the first [35] and second [45] CGI criteria match for it.

\subsubsection{Comparison with SSB}

The model with one massive and no massless gauge boson we have been working with can obviously be obtained by SSB of an $U(1) \simeq O(2)$ gauge model. Let us employ instead of $\eta$ the complex field $\Phi:=i B+m / \kappa+\varphi$. The real part of $\Phi$ is interpreted as a shifted higgs-like field $H=1 / \kappa C_{5}+$ $\varphi=m / \kappa+\varphi$, and we rewrite (29) in terms of it, obtaining the quartic polynomial

$V(\Phi)=V_{0}-\frac{\mu^{2}}{2} \Phi^{t} \Phi+\frac{\lambda}{4}\left(\Phi^{t} \Phi\right)^{2}=: V_{0}+V_{\bmod }(\Phi)$,

where

$$
V_{0}=\frac{m_{H}^{2} m^{2}}{8 \kappa^{2}} ; \quad \mu=\frac{m_{H}}{\sqrt{2}} ; \quad \lambda=\frac{\kappa^{2} m_{H}^{2}}{2 m^{2}} .
$$

In order to extract the SSB model from this, drop the constant term-this has "only" epistemological and gravitycosmological consequences [66]. Then seek the minimum of the potential $\delta V / \delta \Phi=\left(-\mu^{2}+\lambda \Phi^{t} \Phi\right) \Phi=0$. Any solution of this can be "rotated" to a real value $\langle\Phi\rangle=\mu / \sqrt{\lambda}=$ $m / \kappa=: v$. Patently we have reconstructed the "Abelian Higgs model", in which an initially massless vector boson $A$ is held to acquire the mass $m=\kappa v$. (A pity that we cannot switch off the interaction to see whether $A$ was indeed massless.) The remaining scalar particle $\varphi$, or higgs, corresponding to the perturbation of $\Phi$ with respect to $v$, has a mass $\sqrt{2 \lambda} v$, which is precisely $m_{H}$.

\section{2 $S U(2)$ models with only one higgs}

With three gauge fields, the only relevant Lie algebra entering the game is $S U(2)$; this means we take $f_{a b c}=\varepsilon_{a b c}$, whereupon total antisymmetry implies the Jacobi identity. This is surely an important case.

1. The case $m_{1}=m_{2}=m_{3}=0$ is certainly possible, and then neither higgses nor Stückelberg fields are necessary.

2. We see from (15) that if $m_{3}=0$ then $m_{1}=m_{2}$ must hold; the pattern $m_{2}=m_{3}=0, m_{1} \neq 0$ is downright forbidden.

3. The case $m_{3}=0, m_{1}=m_{2} \neq 0$, after the necessary checks of all CGI tree-level conditions, turns out to be possible with one higgs-like field.

4. Finally, if we assume that all masses are different from zero, then necessarily $m_{1}=m_{2}=m_{3}$. This last case, also after all necessary checks, turns out to be possible as well with one higgs-like field.

Physically, the two cases just mentioned correspond respectively to the Georgi and Glashow "electroweak" theory without neutral currents; and to the $S U(2)$ Higgs-Kibble model. Both can be thought of as a limit of the SM, in the second case by setting the Weinberg angle equal to zero and dropping the decoupled photon field.

With respect to the pending checks, let us show first why $m_{1}=m_{2}=m_{3}$ must hold when there are three massive gauge fields. Indeed, (22) implies

$$
\begin{aligned}
4 m_{a}^{2} m_{b}^{2} m_{c}^{2} C_{5}^{2}= & \left(\varepsilon_{a b c}\right)^{2}\left[\left(m_{a}^{2}+m_{b}^{2}+m_{c}^{2}\right)^{2}\right. \\
& \left.-4\left(m_{a}^{2} m_{b}^{2}+m_{c}^{4}\right)\right],
\end{aligned}
$$

where $(a, b, c)$ is any permutation of $(1,2,3)$. Therefore,

$m_{1}^{2} m_{2}^{2}+m_{3}^{4}=m_{2}^{2} m_{3}^{2}+m_{1}^{4}=m_{3}^{2} m_{1}^{2}+m_{2}^{4}$.

This yields

$$
\begin{aligned}
& \left(m_{1}^{2} m_{2}^{2}+m_{3}^{4}\right)-\left(m_{2}^{2} m_{3}^{2}+m_{1}^{4}\right) \\
& \quad=\left(m_{3}^{2}-m_{1}^{2}\right)\left(m_{1}^{2}-m_{2}^{2}+m_{3}^{2}\right)=0, \\
& \left(m_{2}^{2} m_{3}^{2}+m_{1}^{4}\right)-\left(m_{3}^{2} m_{1}^{2}+m_{2}^{4}\right) \\
& \quad=\left(m_{1}^{2}-m_{2}^{2}\right)\left(m_{2}^{2}-m_{3}^{2}+m_{1}^{2}\right)=0,
\end{aligned}
$$


whose only all-positive solution is $m_{1}=m_{2}=m_{3}=: m$; and then $4 m^{6} C_{5}^{2}=m^{4}$ imposes $C_{5}=1 / 2 m$. Formula (16) is void here; the test (20) is cleared as well. This model has been exhaustively studied in [31] and [49]. Note that actually $L_{1}^{2}=0=L_{2}^{3}$ for it.

For the other MVB model with $m_{3}=0,(16)$ is clearly verified for all values of $(a, b)$. As noted earlier, the equality $m_{1}=m_{2}$ can already be deduced from (15), or from (16) alone. Now we find $C_{5}=1 / \mathrm{m}$.

In both $S U(2)$ cases with massive gauge bosons, the couplings are completely determined from CGI, without SSB playing any role. It is nevertheless quite easy to identify the corresponding models in the framework of the Higgs mechanism, with the help of the $S$-matrices. For the three-boson model with one vanishing mass, the results for $f^{3}$ and $f^{5}$ here give

$$
\begin{aligned}
S^{1} & =\left(\begin{array}{ccc}
0 & 0 & -1 \\
0 & 0 & 0 \\
1 & 0 & 0
\end{array}\right) ; \quad S^{2}=\left(\begin{array}{ccc}
0 & 0 & 0 \\
0 & 0 & -1 \\
0 & 1 & 0
\end{array}\right) ; \\
S^{3} & =\left(\begin{array}{ccc}
0 & -1 & 0 \\
1 & 0 & 0 \\
0 & 0 & 0
\end{array}\right),
\end{aligned}
$$

and clearly (24) holds. For the three-boson model with three equal masses, suppressing some null entries in the notation, we likewise get

$$
\begin{aligned}
& S^{1}=\left(\begin{array}{cccc} 
& & 0 & -\frac{1}{2} \\
& & -\frac{1}{2} & 0 \\
0 & \frac{1}{2} & & \\
\frac{1}{2} & 0 & &
\end{array}\right) ; \\
& S^{2}=\left(\begin{array}{cccc} 
& & \frac{1}{2} & 0 \\
& & 0 & -\frac{1}{2} \\
-\frac{1}{2} & 0 & & \\
0 & \frac{1}{2} & &
\end{array}\right) ; \\
& S^{3}=\left(\begin{array}{cccc}
0 & -\frac{1}{2} & & \\
\frac{1}{2} & 0 & & \\
& & 0 & -\frac{1}{2} \\
& & \frac{1}{2} & 0
\end{array}\right) .
\end{aligned}
$$

$5.3 S U(n)$ models with only one higgs

For $S U(3)$ take the basis of Gell-Mann matrices $T_{a}=\lambda_{a} / 2$, for $a=1, \ldots, 8$, normalized by $\operatorname{tr}\left(T_{a} T_{b}\right)=\frac{1}{2} \delta_{a b}$. The wellknown structure constants, defined by $\left[T_{a}, T_{b}\right]=i f_{a b c} T_{c}$, are

$$
f_{123}=1 ; \quad f_{147}=f_{246}=f_{257}=f_{345}=\frac{1}{2} ;
$$

$f_{156}=f_{367}=-\frac{1}{2} ; \quad f_{458}=f_{678}=\frac{\sqrt{3}}{2}$,

and $f_{a b c}=0$ in all cases not arising from these by permuting indices.

It is instructive to play with different mass patterns.

(i) Does the set of constraints allow an $S U(3)$ model with all masses positive? If $a \neq b$ and $f_{a b k} \neq 0$ for exactly one value of $k$, then (22) simplifies to

$$
\begin{aligned}
4 m_{a}^{2} m_{b}^{2} m_{k}^{2} C_{5}^{2}= & \left(f_{a b k}\right)^{2}\left[\left(m_{a}^{2}+m_{b}^{2}+m_{k}^{2}\right)^{2}\right. \\
& \left.-4\left(m_{a}^{2} m_{b}^{2}+m_{k}^{4}\right)\right],
\end{aligned}
$$

and, just as in Sect. 5.2, invariance of $m_{a}^{2} m_{b}^{2}+m_{k}^{4}$ under permutations of $a, b, k$ implies that $m_{a}=m_{b}=m_{k}$. Applying this procedure for $(a, b, k)=(1,2,3),(1,4,7),(2,4,6)$ and $(2,5,7)$ shows that $m_{1}=m_{2}=m_{3}=m_{4}=m_{5}=m_{6}=m_{7}$. However, it should be noted that the cases $(a, b)=(1,2)$ and $(1,4)$ respectively lead to

$4 m_{1}^{4}\left(f_{11}^{5}\right)^{2}=\left(f_{123}\right)^{2} m_{1}^{4}=m_{1}^{4}$

$4 m_{1}^{4}\left(f_{11}^{5}\right)^{2}=\left(f_{147}\right)^{2} m_{1}^{4}=\frac{1}{4} m_{1}^{4}$.

Therefore the inequality $f_{123} \neq \pm f_{147}$ yields an impossibility: there is no all-massive $S U(3)$ model within our approach. Note that $S U(2)$ escapes this sentence because all squared structure constants are equal. The reader should be able to check that the same phenomenon raises obstructions to several other putative $S U(3)$ models.

(ii) This leads us to ponder the "natural" pattern:

$m_{1}=m_{2}=m_{3}=0 ; \quad m_{4}, m_{5}, m_{6}, m_{7} \neq 0$.

Indeed, the "photons" $m_{1}, m_{2}$ force $m_{4}=m_{5}=m_{6}=m_{7}=$ : $m \neq 0$ through use of (22). Then $f_{44}^{5}=\frac{1}{2}$ by just considering in this equation $(a, b)=(4,6)$, say. By considering $(a, b)=(4,5)$, one obtains $m_{8}=2 \mathrm{~m} / \sqrt{3}$, and after some work, it is checked that there is no contradiction in this. Note that $(a, b, k)=(4,5,8)$ is not symmetrical with $(a, b, k)=(4,8,5)$, since in one case there is a massless contribution $\left(f_{453}=\frac{1}{2}\right)$, but not in the other. In conclusion: the model

$m_{1}=m_{2}=m_{3}=0 ; \quad m_{4}=m_{5}=m_{6}=m_{7}=m ;$

$m_{8}=\frac{2}{\sqrt{3}} m \neq 0$

solves the CGI mass conditions (15) and (20).

Turning to $S U(4)$, we can take basis matrices $\left\{T_{a}\right\}$ extending those of $S U(3)$, filled out with a fourth row and column of zeroes, by $\left\{T_{9}, \ldots, T_{15}\right\}$, where

$T_{15}=\frac{1}{2 \sqrt{6}} \operatorname{diag}(1,1,1,-3)$, 
so that $\left\{T_{3}, T_{8}, T_{15}\right\}$ spans the Cartan subalgebra of diagonal matrices, and the off-diagonal ones are the hermitian matrices given in terms of the matrix units $e_{i j}$ by $^{7}$

$$
\begin{aligned}
& T_{1}+i T_{2}=e_{12}, \quad T_{4}+i T_{5}=e_{13}, \quad T_{6}+i T_{7}=e_{23}, \\
& T_{9}+i T_{10}=e_{14}, \quad T_{11}+i T_{12}=e_{24}, \\
& T_{13}+i T_{14}=e_{34} \text {. }
\end{aligned}
$$

We keep the normalization $\operatorname{tr}\left(T_{a} T_{b}\right)=\frac{1}{2} \delta_{a b}$. The structure constants $f_{a b c}$ have the following nonzero squares, with a hexadecimal labelling:

$$
\begin{aligned}
& \left(f_{123}\right)^{2}=1 ; \quad\left(f_{458}\right)^{2}=\left(f_{678}\right)^{2}=\frac{3}{4} ; \\
& \left(f_{89 A}\right)^{2}=\left(f_{8 B C}\right)^{2}=\frac{1}{12} ; \quad\left(f_{8 D E}\right)^{2}=\frac{1}{3} ; \\
& \left(f_{147}\right)^{2}=\left(f_{156}\right)^{2}=\left(f_{19 C}\right)^{2}=\left(f_{1 A B}\right)^{2}=\left(f_{246}\right)^{2}=\frac{1}{4}, \\
& \left(f_{257}\right)^{2}=\left(f_{29 B}\right)^{2}=\left(f_{2 A C}\right)^{2}=\left(f_{345}\right)^{2}=\left(f_{367}\right)^{2}=\frac{1}{4}, \\
& \left(f_{39 A}\right)^{2}=\left(f_{3 B C}\right)^{2}=\left(f_{49 E}\right)^{2}=\left(f_{4 A D}\right)^{2}=\left(f_{59 D}\right)^{2}=\frac{1}{4}, \\
& \left(f_{5 A E}\right)^{2}=\left(f_{6 B E}\right)^{2}=\left(f_{6 C D}\right)^{2}=\left(f_{7 B D}\right)^{2}=\left(f_{7 C E}\right)^{2}=\frac{1}{4} ; \\
& \left(f_{9 A F}\right)^{2}=\left(f_{B C F}\right)^{2}=\left(f_{D E F}\right)^{2}=\frac{2}{3} .
\end{aligned}
$$

Naturally, the structure constants for the Lie subalgebra $S U(3)$ are a subset of those for $S U(4)$. Thus objections to putative models for $S U(3)$ carry over to the $S U(4)$ case. Nevertheless, the allowable pattern for $S U(3)$ given by (33) does have an analogue for $S U(4)$. Let us assume that the bosons labelled by the $S U(3)$ subalgebra are massless, and that the new ones are massive:

$m_{1}=\cdots=m_{8}=0 ; \quad m_{9}, \ldots, m_{15} \neq 0$.

The relation (16) together with (34) gives at once

$m_{9}=m_{10}=m_{11}=m_{12}=m_{13}=m_{14}=: m$,

but remains silent about $m_{15}$. Now we check this for consistency with (22). For $a \leq 8, b \geq 9$, this relation always reduces to $0=\left(\left(2 m^{2}\right)^{2}-4 m^{4}\right) / m^{2}$. Taking $a \neq b$ in the range $\{9, \ldots, 14\}$ we typically obtain

$$
4 m^{2}\left(f_{a a}^{5}\right)^{2}=4 m^{2} \sum_{d \leq 8}\left(f_{a b d}\right)^{2}+\left(f_{a b F}\right)^{2}\left(4 m^{2}-3 m_{15}^{2}\right) .
$$

\footnotetext{
${ }^{7}$ In the standard notation for root vectors, the simple roots $\alpha, \beta, \gamma$ give $E_{\alpha}=e_{12}, E_{\beta}=e_{23}, E_{\gamma}=e_{34}, E_{\alpha+\beta}=e_{13}, E_{\beta+\gamma}=e_{24}, E_{\alpha+\beta+\gamma}=$ $e_{14}$. Note that $\alpha+\gamma$ is not a root of $S U(4)$ since $\left[E_{\alpha}, E_{\gamma}\right]=0$.
}

In most cases, this reduces to $\left(f_{a a}^{5}\right)^{2}=\frac{1}{4}$. When $(a, b)=$ $(9,10)$ or $(11,12)$ or $(13,14)$, we then get

$m^{2}=\frac{1}{3}\left(4 m^{2}\right)+\frac{2}{3}\left(4 m^{2}-3 m_{15}^{2}\right)$,

that is, $3 m^{2}=2 m_{15}^{2}$. When $a=15$ and $9 \leq b \leq 14$, the constraint (22) becomes $4 m^{2}\left(f_{F F}^{5}\right)^{2}=\left(2 m_{15}^{4} / 3 m^{2}\right)=m_{15}^{2}$, consistent with $f_{F F}^{5} / f_{b b}^{5}=m_{15} / m$, as expected. To sum up, this mass pattern is compatible with the CGI mass conditions (15) and (20), provided that

$m_{15}=\sqrt{3 / 2} m$.

No other pattern for $S U(4)$ with one physical scalar seems to solve (15) and (20), although an exhaustive search has not been performed.

Going to the general $S U(n)$ case, one can show likewise that for a theory with $n^{2}-1$ vector bosons and one physical scalar:

$m_{1}=m_{2}=\cdots=m_{n^{2}-2 n}=0 ;$

$m_{(n-1)^{2}}=\cdots=m_{n^{2}-2}=: m \neq 0$;

$m_{n^{2}-1}=\sqrt{\frac{2(n-1)}{n}} m$.

The "odd man out" corresponds to the last Cartan matrix

$T_{R}=C_{n}=\frac{1}{\sqrt{2 n(n-1)}} \operatorname{diag}(1, \ldots, 1,-(n-1))$,

while the previous ones become massless. With the labels $D=n^{2}-2 n, P=n^{2}-3, Q=n^{2}-2, R=n^{2}-1$, then for $(n-1)^{2} \leq a \leq n^{2}-3$ and $b=a+1$, one finds that ${ }^{8}$

$\sum_{d \leq D}\left(f_{a b d}\right)^{2}=\left(f_{P Q D}\right)^{2}=\frac{n-2}{2 n-2}$,

$\left(f_{a b R}\right)^{2}=\left(f_{P Q R}\right)^{2}=\frac{n}{2 n-2}$.

Thus, the analogue of (35) for the $S U(n)$ case is

$m^{2}=\frac{2(n-2) m^{2}}{n-1}+\frac{n}{2(n-1)}\left(4 m^{2}-3 m_{R}^{2}\right)$,

yielding

$m_{R}^{2}=\frac{2 n-2}{n} m^{2}$.

It seems clear that the masses of the gauge particles organize themselves in $S U(n-1)$ multiplets, concretely the fundamental one and a singlet.

${ }^{8}$ To compute $f_{P Q D}$ and $f_{P Q R}$, just evaluate the commutators $\left[C_{n-1}, e_{n-1, n}\right]$ and $\left[C_{n}, e_{n-1, n}\right]$. 
Thus the translation of our allowed models into the SSB phraseology follows a well-trodden path: in general, a vector representation for $S U(n)$ contains $2 n$ real fields, of which $2 n-1$ are "eaten" to provide the longitudinal components for $2 n-1$ "initially massless" gauge fields, leaving $n^{2}-1-(2 n-1)=(n-1)^{2}-1$ "photons" (corresponding to an $S U(n-1)$ as yet "unbroken" symmetry), and the remaining one is the physical higgs field. In such a framework formula (36) is well known [67, Sect. 84]. Things work out similarly for vector representations of $O(n)$; the $O(3) \simeq S U(2)$ case we have seen already in Sect. 5.2.

In conclusion, the CGI mass conditions (15) and (20) efficiently identify SSB-type models in the vector representation. The reader will have no difficulty in writing the $S$ representations and checking the commutation relations. ${ }^{9}$

\subsection{Causal gauge invariance for $S U(3)$ with fields in the adjoint}

We finally turn to more involved models with the scalar fields in the adjoint representation, to exemplify minimal coupling in the CGI framework for such models and to deal with the alleged clash between CGI and SSB in [46].

Concerning GUT models, (most simply) two irreducible representations of pre-higgs particles are needed for SSB to yield something recognizably akin to the SM; to wit, in [68] the adjoint representation $\mathbf{2 4}$ and the complex fundamental representation 5 of $S U(5)$. Thus the question is whether a model with 24 vector bosons, of which 12 have identical nonzero mass and 12 are massless, passes muster in CGI, allowing for 12 higgs-like fields. Naturally, one should seek to answer the similar question for simpler models first. For $S U(2)$, the model with three vector bosons, two with identical nonzero mass and one massless, together with one higgslike field, has been shown in Sect. 5.2 to pass muster in CGI. For $S U(3)$ there would be in the adjoint representation eight vector bosons, four of which have identical nonzero mass and four are massless, with four higgs-like fields. We take up this case as the simplest proxy for our problem, embarking on this from the opposite end to that of Sect. 5.1: first we recall SSB for this model; then in consonance with [45] we verify BRST invariance for the resulting classical Lagrangian; this proves CGI at tree level. Finally, we check the representation property (24) of the $S$-matrices, and substantiate our claim in Sect. 4.2 that minimal coupling follows from CGI. We deem the exercise important and proceed in fastidious detail.

\footnotetext{
${ }^{9}$ Group theory dictates that the sum of the squared $f_{a a}^{5}$ be equal to the Casimir for the corresponding representations, respectively 2 and $\frac{3}{4}$ for the $S U(2)$ models just above. For every $S U(n)$ model of this type $\left(f^{5}\right)^{2}=\frac{1}{4}$ holds.
}

\subsubsection{BRST invariance of the classical Lagrangian}

An invariant potential for that representation is

$V(\Phi)=-\mu^{2} \operatorname{tr} \Phi^{2}+\lambda\left(\operatorname{tr} \Phi^{2}\right)^{2}$

with $\Phi$ a traceless hermitian $3 \times 3$ matrix variable and $\lambda>0$. (We are not striving for maximum generality here, so we have suppressed a term in $\operatorname{tr} \Phi^{3}$. The usual tr $\Phi^{4}$ term is missing since in this somewhat degenerate case $\operatorname{tr} \Phi^{4}=$ $\frac{1}{2}\left(\operatorname{tr} \Phi^{2}\right)^{2}$ by the Cayley-Hamilton formula.) The pattern of symmetry breaking is $S(U(2) \times U(1))$; a minimum of the potential $V(\Phi)$ is of the form

$\langle\Phi\rangle=v \operatorname{diag}(1 / 2 \sqrt{3}, 1 / 2 \sqrt{3},-1 / \sqrt{3})=v \lambda_{8} / 2$,

where $v$ is to be determined such that $\widetilde{V}(v):=V\left(v \lambda_{8} / 2\right)=$ $-\mu^{2} v^{2} / 2+\lambda v^{4} / 4$ be minimal, see [7] for instance. This gives $v_{\min }^{2}=\frac{\mu^{2}}{\lambda} ; \tilde{V}\left(v_{\min }\right)=-\frac{\mu^{4}}{4 \lambda}$. From now on, we just write $v$ for $v_{\text {min }}$. Also write $A^{\mu} \equiv A_{a}^{\mu} T_{a}, \Phi \equiv \phi_{a} T_{a}, u \equiv$ $u_{a} T_{a}, \tilde{u} \equiv \tilde{u}_{a} T_{a}$, using the Gell-Mann basis. One can easily check that a shifted field $\varphi$ is required only for the $\phi_{8}$ component: $\phi_{8}=v+\varphi$.

The covariant derivative in the adjoint representation is of the form $D^{\mu}=\partial^{\mu}-i \kappa\left[A^{\mu}, \cdot\right]$; in components,

$D_{a b}^{\mu}=\delta_{a b} \partial^{\mu}-\kappa f_{a b c} A_{c}^{\mu}, \quad$ and thus

$$
\begin{aligned}
D^{\mu} \Phi & =D^{\mu}\left(\phi_{e} T_{e}\right)=\partial^{\mu} \phi_{e} T_{e}-i \kappa\left[A_{a}^{\mu} T_{a}, \phi_{b} T_{b}\right] \\
& =\partial^{\mu} \phi_{e} T_{e}+\kappa f_{a b c} A_{a}^{\mu} \phi_{b} T_{c}=\left(\partial^{\mu} \phi_{b}+\kappa f_{a b c} A_{c}^{\mu} \phi_{a}\right) T_{b} \\
& =\left(\delta_{a b} \partial^{\mu} \phi_{b}-\kappa f_{a b c} A_{c}^{\mu} \phi_{b}\right) T_{a}=\left(D^{\mu} \Phi\right)^{\dagger} .
\end{aligned}
$$

The Lagrangian for bosonic scalar fields reads

$L_{\Phi}=\operatorname{tr}(D \Phi \cdot D \Phi)-V(\Phi)$

where $V$ is given by (37) with $\mu^{2} \sim \kappa^{0}$ and $\lambda \sim \kappa^{2}$. To determine the mass spectrum of the gauge fields one collects the mass terms in $\operatorname{tr}(D \Phi \cdot D \Phi)$, with $\phi_{8}$ replaced by $v+\varphi$, with the result that

$$
\begin{aligned}
& \kappa^{2}\left(A_{b} \cdot A_{d}\right) f_{8 a b} f_{8 c d} v^{2} \operatorname{tr}\left(T_{a} T_{c}\right) \\
& \quad=\frac{3 v^{2} \kappa^{2}}{8}\left(A_{4} \cdot A_{4}+A_{5} \cdot A_{5}+A_{6} \cdot A_{6}+A_{7} \cdot A_{7}\right) .
\end{aligned}
$$

Hence $m_{1}^{2}=m_{2}^{2}=m_{3}^{2}=m_{8}^{2}=0$, and

$m^{2}:=m_{4}^{2}=m_{5}^{2}=m_{6}^{2}=m_{7}^{2}=3 \mu^{2} \kappa^{2} / 4 \lambda=3 v^{2} \kappa^{2} / 4$.

The potential $V(\Phi)$ contains a mass term only for the field $\varphi$, namely one can show that $V(\Phi)=V_{0}+\mu^{2} \varphi^{2}+$ (terms trilinear and quadrilinear in the fields), where $V_{0}:=$ $\widetilde{V}\left(v_{\min }\right)$. Hence $\varphi$ is the "polar" higgs field, in the direction 
of symmetry breakdown. The other three higgs fields are massless, they are pseudo-Goldstone bosons in the precise sense of [69]. Next we collect all terms $\sim \kappa^{0}$ in $\operatorname{tr}(D \Phi \cdot D \Phi)$. Since there appears a term

$$
\begin{aligned}
2 \operatorname{tr} & {\left[\left(\partial_{\mu} \phi_{a} T_{a}\right)\left(\kappa f_{8 b c} A_{c}^{\mu} v T_{b}\right)\right] } \\
& =\frac{2 m}{\sqrt{3}} \partial_{\mu} \phi_{b} f_{8 b c} A_{c}^{\mu}=-\frac{2 m}{\sqrt{3}} \partial_{\mu} \phi_{b} f_{a b 8} A_{a}^{\mu},
\end{aligned}
$$

we introduce the notation

$$
B_{a}=\frac{2}{\sqrt{3}} f_{a b 8} \phi_{b}
$$

that is, $B_{4}=\phi_{5}, B_{5}=-\phi_{4}, B_{6}=\phi_{7}, B_{7}=-\phi_{6}$, and $B_{a}=0$ for $a=1,2,3,8$; later we shall see that the $B_{a}$ 's are the Stückelberg fields belonging to the massive vector bosons $A_{a}$ (for $a=4,5,6,7$ ). Therefore the remaining bosonic scalar fields $\varphi_{1} \equiv \phi_{1}, \varphi_{2} \equiv \phi_{2}, \varphi_{3} \equiv \phi_{3}$ are the massless physical higgs fields. With that one obtains

$$
\begin{aligned}
\operatorname{tr}(D \Phi \cdot D \Phi)= & \frac{1}{2} \sum_{a=1,2,3} \partial \varphi_{a} \cdot \partial \varphi_{a}+\frac{1}{2} \sum_{a=4,5,6,7} \partial B_{a} \cdot \partial B_{a} \\
& +\frac{1}{2} \partial \varphi \cdot \partial \varphi+\frac{m^{2}}{2} \sum_{a=4,5,6,7}\left(A_{a} \cdot A_{a}\right) \\
& -m \sum_{a=4,5,6,7} A_{a} \cdot \partial B_{a}+O(\kappa) .
\end{aligned}
$$

We now assemble the last pieces of the Lagrangian. For the $m A \cdot \partial B$ terms to add up to a divergence, choose for the gauge-fixing term in the Feynman gauge

$$
\begin{aligned}
L^{\mathrm{gf}}= & L_{0}^{\mathrm{gf}}=-\frac{1}{2} \sum_{a}\left(\partial \cdot A_{a}+m B_{a}\right)^{2} \\
= & -\frac{1}{2} \sum_{a=1}^{8}\left(\partial \cdot A_{a}\right)^{2}-\frac{m^{2}}{2} \sum_{a=4,5,6,7} B_{a}^{2} \\
& -m \sum_{a=4,5,6,7}\left(\partial \cdot A_{a}\right) B_{a} .
\end{aligned}
$$

Due to $F^{\mu v} \equiv \partial^{\mu} A^{v}-\partial^{v} A^{\mu}-i \kappa\left[A^{\mu}, A^{v}\right]$, the Yang-Mills Lagrangian

$$
L^{\mathrm{YM}}=-\frac{1}{2} \operatorname{tr} F^{\mu v} F_{\mu v}=-\frac{1}{4} F_{a}^{\mu \nu} F_{a \mu \nu}
$$

is of the form $L^{\mathrm{YM}}=L_{0}^{\mathrm{YM}}+\kappa L_{1}^{\mathrm{YM}}+\kappa^{2} L_{2}^{\mathrm{YM}}$.

Next we introduce the BRST transformation and verify classical BRST invariance of the total Lagrangian. ${ }^{10}$

\footnotetext{
${ }^{10}$ The reader might ask: why bother? Are not all models generated by SSB automatically BRST invariant? Surely yes. But there are few dis-
}

For $A^{\mu}$ and $\Phi$ the $s$-operator is given by the infinitesimal gauge transformations

$s A^{\mu}=D^{\mu} u=\partial^{\mu} u-i \kappa\left[A^{\mu}, u\right], \quad s \Phi=i \kappa[u, \Phi]$.

For example, the latter gives

$$
\begin{aligned}
s \varphi_{1}= & -\kappa\left(u_{2} \varphi_{3}-u_{3} \varphi_{2}\right) \\
& -\frac{\kappa}{2}\left(u_{4} B_{6}+u_{7} B_{5}+u_{6} B_{4}+u_{5} B_{7}\right) ;
\end{aligned}
$$

in general, $s \varphi_{a}=O(\kappa)$ for $a=1,2,3$; whereas

$$
\begin{gathered}
s B_{4}=m_{4}+\frac{\kappa}{2}\left(u_{6} \varphi_{1}+u_{1} B_{7}-u_{7} \varphi_{2}+u_{2} B_{6}\right. \\
\left.+u_{3} B_{5}+u_{4} \varphi_{3}+\sqrt{3}\left(u_{4} \varphi+u_{8} B_{5}\right)\right)
\end{gathered}
$$

in general, $s B_{a}=m u_{a}+\mathcal{O}(\kappa)$ for $a=4,5,6,7$; and

$s \varphi=-\frac{\kappa \sqrt{3}}{2} \sum_{a=4,5,6,7} u_{a} B_{a}$.

For the ghost fields $u$, we get $s u=i \kappa / 2[u, u]$, as in the massless case, and for the antighosts $\tilde{u}$,

$s \tilde{u}=-(\partial \cdot A+m B)$.

With that the gauge-fixing term can be written as $L_{\mathrm{gf}}=$ $-\frac{1}{2} \sum_{a}\left(s \tilde{u}_{a}\right)^{2}$, as usual in the Feynman gauge.

For the nilpotence of $s$, we find that $s^{2} A=0$ and $s^{2} u=$ 0 are similar to the well-known massless case. In the Lie superalgebra generated by the $A=A_{a}^{\mu} T_{a}$ and the $u=u_{b} T_{b}$, we can write

$$
\begin{aligned}
s^{2} A= & s(D u)=\partial(s u)-i \kappa[s A, u]-i \kappa[A, s u] \\
= & \frac{i \kappa}{2}([u, \partial u]-[\partial u, u])-\frac{\kappa^{2}}{2}(2[[A, u], u] \\
& -[A,[u, u]]) .
\end{aligned}
$$

Since the bracket is symmetric between expressions of ghost number 1, the first two terms cancel; and the second two terms also cancel by the same symmetry and the Jacobi identity, whereby $[A, \cdot]$ is a derivation:

$[A,[u, u]]=[[A, u], u]+[u,[A, u]]=2[[A, u], u]$.

The same identity takes care of $s^{2} \Phi$ :

$s^{2} \Phi=i \kappa([s u, \Phi]-[u, s \Phi])$

cussions of this in the literature: typically textbooks go at great length into the proof that Yang-Mills theories are BRST invariant, and then resolutely tiptoe around the same question for "hidden local symmetry". Reference [70] furnishes an amusing example. 


$$
=-\frac{\kappa^{2}}{2}([[u, u], \Phi]-2[u,[u, \Phi]])=0 .
$$

Vanishing of $s^{2} \tilde{u}$ is discussed further down.

The BRST transformation of $A^{\mu}$ and $\Phi$ has the form of an infinitesimal gauge transformation of the unshifted field. It follows that $s L^{\mathrm{YM}}=0$ and $s L_{\Phi}=0$. We perform the explicit verification for $L_{\Phi}$ by using:

$$
\begin{aligned}
s(D \Phi)= & \partial(s \Phi)-i \kappa([s A, \Phi]+[A, s \Phi]) \\
= & i \kappa \partial([u, \Phi])-i \kappa[\partial u, \Phi] \\
& +\kappa^{2}(-[[A, u], \Phi]+[A,[u, \Phi]]) \\
= & i \kappa[u, \partial \Phi]+\kappa^{2}[u,[A, \Phi]]=i \kappa[u, D \Phi] .
\end{aligned}
$$

Since $D \Phi=\partial \Phi-i \kappa[A, \Phi]$ has ghost number zero, it follows that

$$
\begin{aligned}
s \operatorname{tr}(D \Phi \cdot D \Phi) & =i \kappa \operatorname{tr}([u, D \Phi] \cdot D \Phi+D \Phi \cdot[u, D \Phi]) \\
& =i \kappa \operatorname{tr}[u, D \Phi \cdot D \Phi]=0 .
\end{aligned}
$$

Next we introduce the ghost Lagrangian, chosen in a such a way that $s L^{\mathrm{gf}}+s L^{\mathrm{gh}}$ is a divergence: one sets

$L^{\mathrm{gh}}=\sum_{a=1}^{8} \partial_{\mu} \tilde{u}_{a} s A_{a}^{\mu}-m \sum_{a=4,5,6,7} \tilde{u}_{a} s B_{a}=L_{0}^{\mathrm{gh}}+\kappa L_{1}^{\mathrm{gh}}$,

which yields indeed $s\left(L^{\mathrm{gf}}+L^{\mathrm{gh}}\right)=\partial \cdot I$, with

$$
I:=-\left(\partial \cdot A_{a}+m B_{a}\right) D_{a b} u_{b}=I_{0}+\kappa I_{1} .
$$

Summing up, the total Lagrangian

$$
L_{\mathrm{tot}}=L^{\mathrm{YM}}+L_{\Phi}+L^{\mathrm{gf}}+L^{\mathrm{gh}}=-V_{0}+L_{0}+\kappa L_{1}+\kappa^{2} L_{2}
$$

is classically BRST invariant since

$s L_{\text {tot }}=\partial \cdot I$.

All terms of $L_{2}$ come from $L^{\mathrm{YM}}+L_{\Phi}$. Notice that

$$
\begin{aligned}
L_{0}= & \sum_{a=1}^{8}\left(-\frac{1}{4}\left(A_{a}^{\mu, \nu}-A_{a}^{\nu, \mu}\right)\left(A_{a \mu, v}-A_{a v, \mu}\right)\right. \\
& \left.-\frac{1}{2} \partial A_{a} \cdot \partial A_{a}\right)+\frac{m^{2}}{2} \sum_{a=4,5,6,7} A_{a} \cdot A_{a} \\
& +\sum_{a=1}^{8} \partial \tilde{u}_{a} \cdot \partial u_{a}-m^{2} \sum_{a=4,5,6,7} \tilde{u}_{a} u_{a} \\
& -m \sum_{a=4,5,6,7} \partial \cdot\left(A_{a} B_{a}\right)+\frac{1}{2}\left(\sum_{p=1,2,3} \partial \varphi_{p} \cdot \partial \varphi_{p}\right.
\end{aligned}
$$

$$
\left.+\sum_{a=4,5,6,7}\left(\partial B_{a} \cdot \partial B_{a}-m^{2} B_{a}^{2}\right)+\partial \varphi \cdot \partial \varphi-2 \mu^{2} \varphi^{2}\right)
$$

contains the divergence term $-m \partial \cdot(A B)$, which is irrelevant for the field equations, but contributes to the BRST current $j_{(0)}$ of the free theory (see below).

We may go back now to nilpotence of $s$. The vanishing of $s^{2} \tilde{u}_{a}$ takes place on-shell: with $S_{\text {tot }}=\int d^{4} x L_{\text {tot }}(x)$, the relation

$s^{2} \tilde{u}_{a}=\frac{\delta S_{\mathrm{tot}}}{\delta \tilde{u}_{a}}=0$

follows from the Euler-Lagrange equations.

The BRST current of the free theory can be computed by the formula:

$j_{(0)}^{\mu}=-\left(\frac{\partial L_{0}}{\partial\left(\partial_{\mu} \varphi_{i}\right)} s_{0} \varphi_{i}+I_{0}^{\mu}\right)$,

where there is a summation over $\varphi_{i}=A_{a}^{\mu}, u_{a}, \tilde{u}_{a}, B_{a}, \varphi_{p}$, $\varphi$. For the present model it comes out that

$$
\begin{aligned}
j_{(0)}^{\mu}= & \left(\partial \cdot A_{a}+m_{a} B_{a}\right) \partial^{\mu} u_{a}-u_{a} \partial^{\mu}\left(\partial \cdot A_{a}+m_{a} B_{a}\right) \\
& -\partial_{\nu}\left(\left(\partial^{\nu} A_{a}^{\mu}-\partial^{\mu} A_{a}^{\nu}\right) u_{a}\right)+u_{a}\left(\square+m_{a}^{2}\right) A_{a}^{\mu},
\end{aligned}
$$

by inserting the explicit expressions in (39) for $I_{0}$ and (41) for $L_{0}$ into (42). As explained in [45], the last two terms do not contribute to the nilpotent charge $Q_{\text {in }}$-which is defined only for on-shell fields. Hence, the superderivation $\left[Q_{\text {in }}, \cdot\right]_{\mp}$ is defined as in [35], and the $B_{a}$ are indeed the Stückelberg fields of this reference.

Since the second CGI criterion [45] is fulfilled, the model is causal gauge invariant at the tree level to all orders.

\subsubsection{Minimal coupling from CGI with fields in the adjoint}

Let us now take stock of the CGI relation (24) for the adjoint $S U(3)$ model. In order to avoid misunderstandings, we remind the reader that each vector boson is entitled to its matrix, but there are no rows or columns corresponding to the massless ones. The labelling of the entries is thus given in the order: 4, 5, 6, 7 (for the MVB) and 1,2,3, 8 (for the "higgses"). That is to say, in the notation of (26), we use the multiplet

$$
\eta=\left(\begin{array}{c}
B_{4} \\
B_{5} \\
B_{6} \\
B_{7} \\
\phi_{1} \\
\phi_{2} \\
\phi_{3} \\
v+\varphi
\end{array}\right) \text { versus }\left(\begin{array}{c}
\phi_{1} \\
\phi_{2} \\
\phi_{3} \\
\phi_{4} \\
\phi_{5} \\
\phi_{6} \\
\phi_{7} \\
\phi_{8}
\end{array}\right)=\left(\begin{array}{c}
\varphi_{1} \\
\varphi_{2} \\
\varphi_{3} \\
-B_{5} \\
B_{4} \\
-B_{7} \\
B_{6} \\
v+\varphi
\end{array}\right)
$$


of the previous subsection. To write down the matrices $S^{a}$, we read off $f_{* * *}^{3}, f_{* * *}^{5}, f_{* * *}^{6}$ from $\kappa f_{a b c}\left(A_{c} \cdot \partial \phi_{b}\right) \phi_{a}$ in $\operatorname{tr}(D \Phi \cdot D \Phi)$; note that (40) contains no further contributions to these cubic coupling coefficients. With that, we obtain

$$
S^{1}=\left(\begin{array}{ccccccccc} 
& & 0 & -\frac{1}{2} & & & & \\
& & \frac{1}{2} & 0 & & & & \\
& & -\frac{1}{2} & & & & & & \\
\frac{1}{2} & 0 & & & & & & \\
& & & & 0 & & & \\
& & & & 0 & -1 & \\
& & & & 1 & 0 & \\
& & & & & & 0
\end{array}\right) .
$$

Here we note at once the new relations: $-2 f_{123}^{6}=2 f_{132}^{6}=$ -1 (so $f^{6}$ is not zero). The contributions in the upper left corner come from $f_{147}^{3}$ and such, that do not vanish. Similarly:

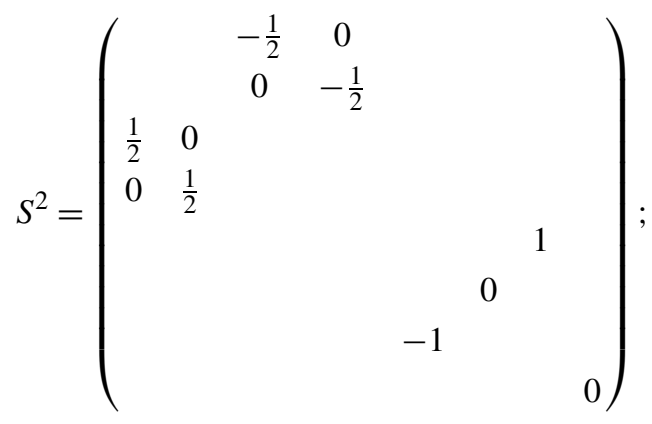

$$
\begin{aligned}
& S^{3}=\left(\begin{array}{cccccccc}
0 & -\frac{1}{2} & & & & & & \\
\frac{1}{2} & 0 & & & & & & \\
& & 0 & \frac{1}{2} & & & & \\
& & -\frac{1}{2} & 0 & & & & \\
& & & & 0 & -1 & & \\
& & & & 1 & 0 & & \\
& & & & & & 0 & \\
& & & & & & & 0
\end{array}\right) ;
\end{aligned}
$$

and the commutation relations of the $S U(2)$ subgroup are clearly fulfilled.

The reader will easily write down the other basis matrices of the $S$-representation and check the group property. The main remark is that, besides the nonvanishing of $f_{1 * *}^{6}$, $f_{2 * *}^{6}, f_{3 * *}^{6}$, there are nondiagonal values of $f_{* * p}^{5}$, for example $f_{472}^{5}=-f_{461}^{5}=\frac{1}{2}$. Matters work in the same way as in this example for any $S U(n)$ model in the adjoint representation for higher $n$. Look no farther for the solution to the conundrum raised by [46]: the model coming by SSB of the representation $\mathbf{2 4}$, responsible for "superstrong breaking" in the original GUT by Georgi and Glashow, is incompatible with the obstructions given by Scharf [35]; but CGI does not exclude it.

We shall now verify that the scalar-gauge Lagrangian resulting from the first CGI method can be expressed as the minimal coupling $\frac{1}{2}(D \eta)^{t} \cdot D \eta=\frac{1}{2} \sum_{b}(D \eta)_{b} \cdot(D \eta)_{b}$. The former can be expressed by the term

$\operatorname{tr}(D \Phi \cdot D \Phi)=\frac{1}{2} \sum_{b}(D \phi)_{b} \cdot(D \phi)_{b}$

$(D \phi)_{b}:=\partial \phi_{b}-\kappa f_{b a c} A_{c} \phi_{a}$

obtained by SSB, since construction of the $S U(3)$-adjoint model by SSB agrees with what one obtains by CGI (as shown in Sect. 5.4). Then $\frac{1}{2}(D \eta)^{t} \cdot D \eta=\operatorname{tr}(D \Phi \cdot D \Phi)$ follows from noting that the sets of covariant derivatives agree. For example, by direct calculation,

$$
\begin{aligned}
(D \phi)_{1}= & \partial \varphi_{1}+\kappa\left(A_{2} \varphi_{3}-A_{3} \varphi_{2}\right) \\
& +\frac{\kappa}{2}\left(A_{4} B_{6}+A_{6} B_{4}+A_{5} B_{7}+A_{7} B_{5}\right)=(D \eta)_{5}, \\
(D \phi)_{4}= & -\partial B_{5}-\frac{\kappa}{2}\left(A_{1} B_{6}-A_{2} B_{7}+A_{3} B_{4}-A_{5} \varphi_{3}\right. \\
& \left.-A_{6} \varphi_{2}-A_{7} \varphi_{1}\right)-\frac{\kappa \sqrt{3}}{2}\left(-A_{5}(\varphi+v)+A_{8} B_{4}\right) \\
= & -(D \eta)_{2}, \\
(D \phi)_{8}= & \partial \varphi+\frac{\kappa \sqrt{3}}{2}\left(A_{4} B_{4}+A_{5} B_{5}+A_{6} B_{6}+A_{7} B_{7}\right) \\
= & (D \eta)_{8} .
\end{aligned}
$$

Agreement of other components follows by permutation.

It is instructive to write down term by term the equality $\frac{1}{2}(D \eta)^{t} \cdot D \eta=$ scalar gauge Lagrangian obtained by the first CGI method, see (27). The validity of the resulting equations is not limited to the $S U(3)$-adjoint model.

The mass terms for vector gauge fields originate from $\left(A_{a} \cdot A_{b}\right) v^{t}\left[S_{a}, S_{b}\right]_{+} v$, where $v^{t}:=\left(v_{1}, \ldots, v_{z}\right)$. Equating the corresponding coefficients, we find that

$$
-\frac{\kappa^{2}}{4} v^{t}\left(-{ }^{t} G^{a} G^{b}-{ }^{t} G^{b} G^{a}+\left[H^{a}, H^{b}\right]_{+}\right) v=\delta_{a b} m_{a}^{2}
$$

For the $S U(3)$-adjoint model, with the scalar multiplet as in (43), on inserting the above $S$-matrices, this formula gives indeed $\frac{3}{8} v^{2} \kappa^{2} \sum_{k=4}^{7} A_{k} \cdot A_{k}=\frac{1}{2} m^{2} \sum_{k=4}^{7} A_{k} \cdot A_{k}$, like in (38). Note that although $f^{6} \neq 0$, it does not contribute.

By the definition of the $S$-matrices, the couplings $L_{1}^{3}+$ $L_{1}^{5}+L_{1}^{6}$ must be contained in $\left(A_{a} \cdot \partial \eta^{t}\right) S^{a} \eta-\eta^{t} S^{a}\left(A_{a} \cdot \partial \eta\right)$. Indeed, we obtain

$$
\begin{aligned}
& \left(A_{a} \cdot \partial \eta^{t}\right) S^{a} \eta-\eta^{t} S^{a}\left(A_{a} \cdot \partial \eta\right) \\
& \quad=L_{1}^{3}+L_{1}^{5}+L_{1}^{6}-\kappa f_{a b p}^{5}\left(A_{a} \cdot \partial B_{b}\right) v_{p} .
\end{aligned}
$$


The additional $(A \cdot \partial B)$ term gives, as expected,

$$
\begin{aligned}
-\kappa f_{a b p}^{5}\left(A_{a} \cdot \partial B_{b}\right) v_{p} & =-\kappa v f_{a b 8}^{5}\left(A_{a} \cdot \partial B_{b}\right) \\
& =-\frac{\sqrt{3} \kappa v}{2} \sum_{a: m_{a} \neq 0}\left(A_{a} \cdot \partial B_{a}\right) \\
& =-m \sum_{a: m_{a} \neq 0}\left(A_{a} \cdot \partial B_{a}\right) .
\end{aligned}
$$

Moreover, in $\left(A_{a} \cdot A_{b}\right) \eta^{t}\left[S_{a}, S_{b}\right]_{+} \eta$ there are trilinear terms corresponding to $L_{1}^{2}+L_{1}^{7}$. Equating the pertinent coefficients, we get

$$
\begin{aligned}
& f_{a b \star}^{2}=-\frac{\kappa^{2}}{2}\left(F^{a} G^{b}+F^{b} G^{a}+G^{a} H^{b}+G^{b} H^{a}\right) v ; \\
& f_{a b \star}^{7}=\frac{\kappa^{2}}{2}\left({ }^{t} G^{a} G^{b}+{ }^{t} G^{b} G^{a}-H^{a} H^{b}-H^{b} H^{a}\right) v .
\end{aligned}
$$

The pattern exemplified above is typical for $S U(n)$ models with the scalar fields in the adjoint representation. An even simpler example is provided by the three-boson case with two identical masses and one "photon" of Sect. 5.2. Proceeding as above, the reader should have no difficulty in verifying that $\frac{1}{2}(D \eta)^{t} \cdot D \eta$ equals the SSB-type expression $\operatorname{tr}(D \Phi \cdot D \Phi)$.

\section{Conclusion}

The Higgs sector of the SM, in the perspective of basic structures of gauge theories, plays a somewhat ambiguous and enigmatic role. The massless and massive gauge bosons which are the carriers of the fundamental forces belong to what might be termed radiation, in analogy to electrodynamics. Now, by itself, the gauge boson sector of gauge theories of interest for physics defines a nontrivial theory. Quarks and leptons, on the other hand, belong to the category matter which cannot "live on its own" without the gauge sector. Indeed, a theory of quarks and leptons only is a theory of free particles and, being untestable in experiment, is uninteresting. The Higgs sector's place in this classification is perhaps not as obvious as it may appear at first sight. Extensions of the Standard Model within noncommutative geometry [71-74] view scalar fields as an integral part of the connection, i.e. classify them in the sector of gauge bosons and hence place them in the category "radiation". Its alleged phenomenological role of providing masses for the fermions and some bosons of the model, and its likely kinship with dark matter [15], in turn, might suggest that it rather represents another form of "matter" beyond the ordinary one made out of quarks and leptons.

Be that as it may, the traditional description of the Higgs sector by means of the "hidden symmetry" concept, however attractive it may seem from the standpoint of group theory, is still a purely classical one. Classical and semi-classical mechanisms have their uses in quantum field theory: no one will dispute that anomalies are a quantum phenomenon although they can be described in purely classical terms [75, 76]. For conceptual clarity, nevertheless, one should cling to root quantum explanations.

One may reckon, furthermore, that on the subject of this paper the panorama has been obscured by much theoretical prejudice. The Higgs mechanism is burdened with giving masses to all matter and force fields; a heavy load to carry indeed. Explicit mass terms for the vector bosons of electroweak theory are said to be forbidden by gauge invariance. It is not so: these mass terms can be accommodated in gauge theory by regarding the "swallowed" Higgs ghosts of lore as Stuickelberg fields. Also it is said that chirality of the fermions and gauge invariance in weak interactions requires the Higgs mechanism to generate masses by Yukawa couplings. It is not so: one can use Dirac masses for the fermions and derive chirality of couplings from causal gauge invariance $[41,42]$.

In conclusion, starting from the BRST description for MVB as fundamental objects [27, 31, 33, 35, 41], we have perturbatively performed a second reality check of the Higgs mechanism in the spirit of causal gauge invariance, with the outcome that, reversing the dictum by Yang, interaction dictates symmetry-fixing the models up to minute details. ${ }^{11}$ That vindicates the conclusions of the historically first reality check $[29,30]$ as well. Beyond reestablishing the manifold aspects of renormalizable gauge theories, the analysis in the path-breaking book [35] has been completed with the causal derivation of minimal coupling. This allows now for a reliable list of renormalizable couplings in BRST-invariant models. The contention that there might be contradiction between causal gauge invariance and some GUT models [46] has been laid to rest.

Acknowledgements MD was supported by the Deutsche Forschungsgemeinschaft through the Institutional Strategy of the University of Göttingen. JMG-B is grateful to Luis J. Boya for calling attention to the paper [79] and sound counsel on representations of the classical Lie algebras. He is also indebted to Jean Zinn-Justin and Marc Henneaux for illuminating conversations on the BRST invariance of SSB models. His work was supported by DGIID-DGA (grant E24/2). FS thanks Stefan Tapprogge for valuable information about the experimental Higgs searches. JCV acknowledges support from the Vicerrectoría de Investigación of the University of Costa Rica.

\section{Appendix A: On the Standard Model in CGI}

Postulating four gauge bosons, one of which is massless, and one physical scalar, one is unerringly led by CGI [41] to

\footnotetext{
${ }^{11}$ A role for MVB as sources of symmetry, with very different intent, is found in [77].
} 
$U(2)$ symmetry $^{12}$ and the "phenomenological" boson sector of the SM. The only alternatives allowed by CGI are limits of the SM in which one, three or all of the vector particles decouple. In standard presentations the $U(2)$ symmetry is said to be "broken", among other reasons, because there is only one conserved quantity, electric charge, instead of four. But from our viewpoint symmetry is broken at the level of the free Lagrangian, due to different masses (the residual equality of two masses reflects conservation of electric charge). This is to say that there is a natural basis of the Lie algebra linked to the pattern of masses. The role of the constraint (20) is precisely to pick out this basis. Little support comes from this quarter for the idea that the SM as it stands is "imperfectly unified".

Now, the CGI conditions can likewise be applied to the fermion sector. As hinted at earlier, incompatibility of Dirac masses for fermions with gauge symmetry is just another popular misconception. The basic fermion-vectorboson vertices between carriers and matter in a gauge theory are written $\kappa\left(b^{a} \bar{\psi} A_{a} \psi+b^{\prime a} \bar{\psi} A_{a} \gamma^{5} \psi\right)$, à la Bjorken and Drell, with $\bar{\psi}$ the Dirac adjoint spinor and $b, b^{\prime}$ appropriate coefficients. Taking for the fermions the known ones-see [41, 42] and [35, Sect. 4.7]—-first-order gauge invariance already determines some couplings: in particular, the photon has no axial vector couplings "because" there is no Stückelberg field for it. At second order, contractions between the corresponding fermionic $Q$-vertex and the bosonic $L_{1}$ and between the bosonic $Q$-vertex and $L_{1}^{F}$ determine the matter couplings completely (contractions between $L_{1}^{F}$ and its $Q$ vertex contribute nothing). It is beautiful to behold that couplings of the physical scalar to fermions are proportional to their mass, and that chirality of the interactions need not to be brought from the outside, but is a consequence of CGI. As usual, CGI at third order for tree graphs fixes the higgs potential [42]. Since the causal version of the SM leads to the same phenomenological Lagrangian, excepting only that the vacuum expectation value of the higgs field is zero, there is no way within pure particle physics to tell it apart from the ordinary version. However, all the above springs just from the BRST treatment for free spin one bosons and causal renormalization theory. This stands our approach in good stead in the face of breakdown of symmetry.

\section{Appendix B: Derivation of the second main constraint}

The three constraints (25) are crucial in this paper. All of them follow from (8), that is, CGI for second-order tree diagrams by using the technique explained in Sect. 2.2. The third constraint is derived like the first, except that the roles

\footnotetext{
${ }^{12}$ As remarked early on in [78], the true group of the electroweak interaction is $U(2)$, not $S U(2) \times U(1)$.
}

of the $B$ - and $\varphi$-fields are reversed; and for the first one no substantial deviation from reference [35] is required. Thus we focus on the second constraint.

Since only those terms of $P^{v}$ having a derivative $\partial^{v}$ on a field operator contribute, we list only such terms:

$$
\begin{aligned}
P^{v}= & \kappa\left(f_{a b c}\left(-A_{\mu a} u_{b} \partial^{v} A_{c}^{\mu}+\frac{1}{2} u_{a} u_{b} \partial^{v} \tilde{u}_{c}\right)\right. \\
& -2 f_{a b c}^{3} u_{a} B_{b} \partial^{v} B_{c}-f_{a b p}^{5} u_{a}\left(B_{b} \partial^{v} \varphi_{p}-\partial^{v} B_{b} \varphi_{p}\right) \\
& \left.-2 f_{a p q}^{6} u_{a} \varphi_{p} \partial^{v} \varphi_{q}+\cdots\right) .
\end{aligned}
$$

See (4.3.17) in [35] in this respect. The $k$-th term in this expression will be called $P_{k}(k=1, \ldots, 6)$ henceforth. We omit the notation for normal ordering.

The second constraint is obtained from (8) by equating the coefficients of $\delta(x-y) u_{a} u_{b} \tilde{u}_{d} \varphi_{p}$.

A type 1 term $N_{2}=C_{a b d p} \delta B_{a} u_{b} \tilde{u}_{d} \varphi_{p}$ would contribute. However, as indicated in Sect. 4.2, there are no quartic terms containing ghost fields $u, \tilde{u}$. Also, there are no type 2 or type 4 terms, since the contributions of these terms are $\sim\left(\partial \phi_{1}\right) \phi_{2} \phi_{3} \phi_{4}$ and not $\sim \phi_{1} \phi_{2} \phi_{3} \phi_{4}$.

The following type 3 terms do contribute. For the contraction of $\partial \tilde{u}$ in $P_{2}$ with $u$ in $L_{1}^{8}$, we must be careful with the sign, because of the many Fermi operators. In the region $x^{0}>y^{0}$ we obtain

$$
\begin{aligned}
& T_{2}\left(P_{2}^{v}(x) L_{1}^{8}(y)\right) \\
& \quad=P_{2}^{v}(x) L_{1}^{8}(y) \sim: u_{a} u_{b} \partial^{v} \tilde{u}_{c}(x):: \tilde{u}_{d} u_{c^{\prime}} \varphi_{p}(y): \\
& =-i \delta_{c c^{\prime}} \partial_{x}^{v} \Delta_{m}^{+}(x-y): u_{a}(x) u_{b}(x) \tilde{u}_{d}(y) \varphi_{p}(y):+\cdots
\end{aligned}
$$

and for $y^{0}>x^{0}$,

$$
\begin{aligned}
T_{2} & \left(P_{2}^{v}(x) L_{1}^{8}(y)\right) \\
& =L_{1}^{8}(y) P_{2}^{v}(x) \sim: \tilde{u}_{d} u_{c^{\prime}} \varphi_{p}(y):: u_{a} u_{b} \partial^{v} \tilde{u}_{c}(x): \\
& =-i \delta_{c c^{\prime}} \partial_{x}^{v} \Delta_{m}^{+}(y-x): u_{a}(x) u_{b}(x) \tilde{u}_{d}(y) \varphi_{p}(y):+\cdots
\end{aligned}
$$

by using Wick's theorem. Together these give a term

$$
\begin{aligned}
\sim & -i \partial_{x}^{v}\left(\Delta_{m}^{+}(x-y) \theta\left(x^{0}-y^{0}\right)+\Delta_{m}^{+}(y-x) \theta\left(y^{0}-x^{0}\right)\right) \\
& =-i \partial^{v} \Delta_{m}^{F}(x-y) .
\end{aligned}
$$

On computing the divergence $\partial_{v}^{x}$ and adding the term with $x$ and $y$ exchanged, we find the contribution

$-i f_{a b c} f_{d c p}^{8} u_{a}(x) u_{b}(x) \tilde{u}_{d}(x) \varphi_{p}(x) \delta(x-y)$.

Additional terms come from contracting $\partial \varphi$ in $P_{6}$ with $\varphi$ in $L_{1}^{8}$ and $\partial B$ in $P_{5}$ with $B$ in $L_{1}^{4}$. These respectively read

$$
\begin{aligned}
& -2 i\left(f_{a p v}^{6} f_{d b v}^{8}-f_{b p v}^{6} f_{d a v}^{8}\right) u_{a}(x) u_{b}(x) \tilde{u}_{d}(x) \varphi_{p}(x) \\
& \quad \times \delta(x-y) ;
\end{aligned}
$$




$$
\begin{aligned}
& -i\left(-f_{a k p}^{5} f_{d b k}^{4}+f_{b k p}^{5} f_{d a k}^{4}\right) u_{a}(x) u_{b}(x) \tilde{u}_{d}(x) \varphi_{p}(x) \\
& \quad \times \delta(x-y) .
\end{aligned}
$$

On adding all terms and setting the resulting coefficient equal to zero, the second constraint (25) follows.

\section{Appendix C: Epistemological second thoughts}

Among the motivations of this article was the realization of how relatively poor a reputation SSB enjoys among knowledgeable philosophers of science. In such quarters it is regarded as a non-empirical device of little explanatory value. More precisely, Higgs' argument is rightly seen as possessing tremendous heuristic value in the context of discovery, but less so in the context of justification.

"As the semi-popular presentations put it, "particles get their masses by eating the higgs field.' Readers of Scientific American can be satisfied with these justso stories. But philosophers of science should not be. For a genuine property like mass cannot be gained by eating descriptive fluff, which is just what gauge is. (They) should be asking... what is the objective (i.e., gauge invariant) structure of the world corresponding to the gauge theory presented in the Higgs mechanism?"

This criticism by Earman is quoted in [79], which tries to explore the epistemological meaning of SSB. Consult as well [80]. A final remark is in order. When constructing via CGI the Higgs potentials $V$, a zero vacuum expectation value emerges. Making this explicit is however noxious to the Higgs mechanism interpretation. On which interpretation is preferable, we quote Kibble:

"It is perfectly possible to describe our model without ever introducing the notion of SSB, merely by writing down the (phenomenological) Lagrangian. Indeed if the physical world were described by this model, it is to the latter rather than to the former to which we should be led by experiment. The only advantage of SSB is that it is easier to understand the appearance of an exact symmetry than an approximate one" [81].

Such honesty is nowadays refreshing. It is all perhaps a matter of taste. Tastes change over time, though; and to some the works of the "exact" symmetry are uglier than the refusal to deal with unobservable fields.

\section{References}

1. F. Englert, R. Brout, Phys. Rev. Lett. 13, 321 (1964)

2. P.W. Higgs, Phys. Lett. 12, 132 (1964)
3. P.W. Higgs, Phys. Rev. Lett. 13, 508 (1964)

4. G.S. Guralnik, C.R. Hagen, T.W.B. Kibble, Phys. Rev. Lett. 13, 585 (1964)

5. G.S. Guralnik, Int. J. Mod. Phys. A 24, 2601 (2009)

6. I.J.R. Aitchison, A.J.G. Hey, Gauge Theories in Particle Physics: $Q C D$ and the Electroweak Theory (IOP, Bristol, 2004)

7. L. O'Raifeartaigh, Group Structure of Gauge Theories (Cambridge University Press, Cambridge, 1986)

8. C. Burgess, G. Moore, The Standard Model: A Primer (Cambridge University Press, Cambridge, 2007)

9. M.J.G. Veltman, Rev. Mod. Phys. 72, 341 (2000)

10. M.J.G. Veltman, in The Rise of the Standard Model, ed. by L. Hoddeson, L. Brown, M. Riordan, M. Dresden (Cambridge University Press, Cambridge, 1997), p. 145

11. H. Flächer, M. Goebel, J. Haller, A. Hoecker, K. Mönig, J. Stelzer, Eur. Phys. J. C 60, 543 (2009)

12. M.S. Chanowitz, Phys. Rev. D 66, 073002 (2002)

13. J. Erler, P. Langacker, Acta Phys. Pol. B 39, 2595 (2008)

14. S. Gopalakrishna, S. Jung, J.D. Wells, Phys. Rev. D 78, 055002 (2008)

15. H. Davoudiasl, R. Kitano, T. Li, H. Murayama, Phys. Lett. B 609, $117(2005)$

16. R. Jora, S. Moussa, S. Nasri, J. Schechter, M. Naeem Shahid, Int. J. Mod. Phys. A 23, 5159 (2008)

17. F. Wilczek, in Perspectives on LHC Physics, ed. by G. Kane, A. Pierce (World Scientific, Singapore, 2008)

18. T. Aaltonen et al., arXiv:0810.5357 [hep-ex]

19. F. Ptochos, arXiv:0907.0146 [hep-ex]

20. P. Giromini et al., arXiv:0810.5730 [hep-ph]

21. M.J. Strassler, arXiv:0811.1560 [hep-ph]

22. M.J. Strassler, arXiv:0902.0377 [hep-ph]

23. V.M. Abazov et al., arXiv:1005.2757 [hep-ex]

24. B.A. Dobrescu, P.J. Fox, A. Martin, arXiv:1005.4238 [hep-ph]

25. M. Dütsch, F. Krahe, G. Scharf, Nuovo Cimento A 106, 277 (1993)

26. G. Scharf, Finite Quantum Electrodynamics. The Causal Approach (Springer, Berlin, 1995)

27. L. Alvarez-Gaumé, L. Baulieu, Nucl. Phys. B 212, 255 (1983)

28. S. Pokorski, Gauge Field Theories (Cambridge University Press, Cambridge, 2000)

29. J.M. Cornwall, D.N. Levin, G. Tiktopoulos, Phys. Rev. D 10, 1145 (1974)

30. D.N. Levin, G. Tiktopoulos, Phys. Rev. D 12, 415 (1975)

31. M. Dütsch, B. Schroer, J. Phys. A 33, 4317 (2000)

32. H. Ruegg, M. Ruiz-Altaba, Int. J. Mod. Phys. A 19, 3265 (2004)

33. J.M. Gracia-Bondía, in Geometrical and Topological Methods for Quantum Field Theory, ed. by H. Ocampo, E. Pariguan, S. Paycha (Cambridge University Press, Cambridge, 2010), p. 220

34. P. Costello, arXiv:0905.3570 [math.OA]

35. G. Scharf, Quantum Gauge Theories: A True Ghost Story (Wiley, New York, 2001)

36. M. Dütsch, T. Hurth, F. Krahe, G. Scharf, Nuovo Cimento A 106, 1029 (1993)

37. M. Dütsch, T. Hurth, F. Krahe, G. Scharf, Nuovo Cimento A 107, 375 (1994)

38. M. Dütsch, T. Hurth, G. Scharf, Nuovo Cimento A 108, 679 (1995)

39. M. Dütsch, T. Hurth, G. Scharf, Nuovo Cimento A 108, 737 (1995)

40. T. Hurth, Ann. Phys. (N.Y.) 244, 340 (1995)

41. M. Dütsch, G. Scharf, Ann. Phys. (Leipz.) 8, 359 (1999)

42. A. Aste, G. Scharf, M. Dütsch, Ann. Phys. (Leipz.) 8, 389 (1999)

43. D.R. Grigore, J. Phys. A 33, 8443 (2000)

44. R. Stora, Talk given at ESI, Vienna (1997)

45. M. Dütsch, Ann. Phys. (Leipz.) 14, 438 (2005)

46. M. Ambauen, G. Scharf, hep-th/0409062 
47. H. Epstein, V. Glaser, Ann. Inst. H. Poincaré A 19, 211 (1973)

48. T. Hurth, K. Skenderis, Nucl. Phys. B 541, 566 (1999)

49. M. Dütsch, F.-M. Boas, Rev. Math. Phys. 14, 977 (2002)

50. M. Dütsch, K. Fredenhagen, Commun. Math. Phys. 243, 275 (2003)

51. F. Brennecke, M. Dütsch, Rev. Math. Phys. 20, 119 (2008)

52. A. Burnel, Noncovariant Gauges in Canonical Formalism (Springer, Berlin, 2009)

53. M. Dütsch, K. Fredenhagen, Commun. Math. Phys. 203, 71 (1999)

54. T. Kugo, I. Ojima, Progr. Theor. Phys. Suppl. 66, 1 (1979)

55. G. Barnich, M. Henneaux, T. Hurth, K. Skenderis, Phys. Lett. B 492, 376 (2000)

56. G. Barnich, T. Hurth, K. Skenderis, Phys. Lett. B 588, 111 (2004)

57. P.M. Mathews, M. Seetharaman, M.T. Simon, Phys. Rev. D 9 , 1706 (1974)

58. B. Schroer, Eur. Phys. J. H 35, 53 (2010)

59. B. Schroer, arXiv:1006.3543 [hep-th]

60. A. Burnel, Acta Phys. Pol. B 27, 2441 (1996)

61. M. Chaichian, K. Nishijima, Eur. Phys. J. C 22, 463 (2001)

62. K. Nishijima, A. Tureanu, Eur. Phys. J. C 53, 649 (2008)

63. M. Dine, Supersymmetry and String Theory. Beyond the Standard Model (Cambridge University Press, Cambridge, 2007)

64. M.J.G. Veltman, Acta Phys. Pol. B 25, 1627 (1994)

65. J.M. Gracia-Bondía, arXiv:0809.0160 [hep-th]
66. M.J.G. Veltman, Phys. Rev. Lett. 34, 777 (1975)

67. M. Srednicki, Quantum Field Theory (Cambridge University Press, Cambridge, 2007)

68. H. Georgi, S.L. Glashow, Phys. Rev. Lett. 32, 438 (1974)

69. S. Weinberg, Phys. Rev. Lett. 29, 1698 (1972)

70. S. Weinberg, The Quantum Theory of Fields II (Cambridge University Press, Cambridge, 1996)

71. A. Connes, J. Lott, Nucl. Phys. B, Proc. Suppl. 18, 29 (1990)

72. R. Coquereaux, G. Esposito-Farèse, F. Scheck, Int. J. Mod. Phys. A 7, 6555 (1992)

73. H. Figueroa, J.M. Gracia-Bondía, F. Lizzi, J.C. Várilly, J. Geom. Phys. 26, 329 (1998)

74. A.H. Chamseddine, A. Connes, M. Marcolli, Adv. Theor. Math. Phys. 11, 991 (2007)

75. A. Heil, N.A. Papadopoulos, B. Reifenhäuser, F. Scheck, Nucl. Phys. B 293, 445 (1987)

76. A. Heil, A. Kersch, N.A. Papadopoulos, B. Reifenhäuser, F. Scheck, Ann. Phys. (N.Y.) 200, 206 (1990)

77. J.L. Chkareuli, C.D. Froggatt, H.B. Nielsen, Phys. Rev. Lett. 87, 091601 (2001)

78. F. Scheck, Leptons, Hadrons and Nuclei (North-Holland, Amsterdam, 1983)

79. H. Lyre, Int. Stud. Philos. Sci. 22, 119 (2008)

80. J. Earman, Int. Stud. Philos. Sci. 18, 173 (2004)

81. T.W.B. Kibble, Phys. Rev. 155, 1554 (1967) 